\title{
Research Paper \\ Comparing Impulse Control and Emotional Regulation in Patients With and Without Food Addiction
}

\author{
Shirzad Babaei ${ }^{1}$, Aliasghar Asgharnejad Farid ${ }^{2 *}$, Fahimeh Fathali Lavasani ${ }^{3}$, Behrooz Birashk ${ }^{4}$
}

1. MSc. in Clinical Psychology, Department of Clinical Psychology, School of Behavioral Sciences and Mental Health (Tehran Institute of Psychiatry), Iran University of Medical Sciences, Tehran, Iran.

2. PhD in Clinical Psychology, Associate Professor, Department of Clinical Psychology, School of Behavioral Sciences and Mental Health (Tehran Institute of Psychiatry) Iran University of Medical Sciences, Tehran, Iran.

3. PhD in Clinical Psychology, Assistant Professor, Department of Clinical Psychology, School of Behavioral Sciences and Mental Health (Tehran Institute of Psychiatry) Iran University of Medical Sciences, Tehran, Iran.

4. PhD in Counseling Psychology, Associate Professor, Department of Clinical Psychology, School of Behavioral Sciences and Mental Health (Tehran Institute of Psychiatry), Iran University of Medical Sciences, Tehran, Iran.

\begin{tabular}{l|l}
$\begin{array}{c}\text { Use your device toscan } \\
\text { and read the article online }\end{array}$ \\
Regulation in Patients With Food Addiction and Control Group (Persian)]. Iranian Journal of Psychiatry and Clinical Psychology. \\
2017; 23(1):22-37. https://doi.org/10.18869/nirp.ijpcp.23.1.22 \\
doj: https://doi.org/10.18869/nirp.ijpcp.23.1.22
\end{tabular}

Received: 29 Sep. 2016

Accepted: 08 Jan. 2017

Key words:

Food addiction Impulse control Emotional regulation

\section{A B S TR A C T}

Objectives This study examined the variables of impulse control and emotional regulation in patients with food addiction compared to the control group.

Methods The study was cross-sectional, and the sample was composed of men. Thirty patients admitted to a clinic for food addiction were part of the research group, and another 30 persons were part of the control group. Semi-structured interviews were used for screening the participants. The Yale Food Addiction Scale was used for diagnosis and research groups, and the Barratt Impulsiveness Scale and Graz Difficulties in Emotional Regulation Scale were used to assess impulse control and emotional regulation, respectively. To evaluate each variable, multivariate analysis of variance was conducted.

Results The findings demonstrated that patients had statistically achieved the lowest scores in all aspects of food addiction, impulse control and emotional regulation compared with the control group.

Conclusion Patients with food addiction had high impulsivity and lower emotion regulation compared to the control group. Thus, it can be inferred that impaired impulse control and emotional regulation are important factors in the formation and persistence of addictions.

\section{Extended Abstract}

\section{Introduction}

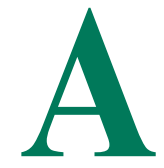

ddiction is an acquired chronic recurrent disorder that starts with some unpleasant experiences and responses. It is associated with a series of engagement, moderation, tolerance, rejection, psychological experiments, and social consequences. In other words, addiction is a destructive tension between drugs (or an activity such as gambling) and patients [1]. As food addiction is a new diagnostic category with only a few research works focusing on it, this paper aims to examine the components of impulse control and emotion regulation in food addiction. Our assumption is that people with food addiction probably register poor performances with reference to the components of impulse control and emotion regulation compared with the control group.

\section{* Corresponding Author:}

Aliasghar Asgharnejad Farid, PhD

Address: Department of Clinical Psychology, School of Behavioral Sciences and Mental Health (Tehran Institute of Psychiatry), Iran University of Medical

Sciences, Tehran, Iran.

Tel: +98 (912) 2979917

E-mail: asgharnejad.ali@gmail.com 


\section{Method}

The research is a causal-comparative and cross-sectional study (ex post facto). Forty-three male patients with food addiction who were referred to the obesity clinic of Imam Khomeini Hospital and obesity clinics in Tehran were initially considered for the study. Of them, 30 men were selected via purposive sampling as the experimental group. Of the 72 employees of the Imam Khomeini Hospital, obesity-treating private clinics in Tehran and students of Tehran University of Medical Sciences, 30 males were selected as the control group according to the simulations carried out by the research group. The exclusion criteria included severe diagnostic psychiatric disorders. A semi-structured interview (SCID-I) was used to screen patients. The Yale Food Addiction questionnaire was used to identify the research groups. The Barratt Impulsiveness Scale and Gratz's Difficulty in Emotional Regulation Scale Questionnaire were used in order to assess impulse control and emotional regulation, respectively. To evaluate each variable, multivariate analysis of variance (MANOVA) (value of statistical significance is 0/05) was used in conjunction with SPSS software version 16.

\section{Results}

We found that 13 patients in the food addiction group (43.3\%) had acquired a diploma compared to 15 patients in the control group (50\%). Equal number of patients, i.e., 8 (26.7\%), had attained an above-diploma education level in both the groups 8 (26.7\%). The number of patients who had obtained a Bachelor's degree in the food addiction group was $9(30 \%)$ while the count in the control group was $7(23.3 \%)$.

The first hypothesis was that the impulse control in people with food addiction is different from that of the control group. To investigate this hypothesis, the multivariate analysis of variance was used. The results showed that there is a difference between the groups with regard to at least one variable. To examine the assumptions of multivariate analysis of variance and data normalization, the Kolmogorov-Smirnov test was used. The results of this test were higher than 0.05 $(\mathrm{P}>0.05)$ in all indices, resulting in data normality Bartlett sphericity test was used to investigate the correlation between the dependent measures. The results indicated that there was a significant correlation $(\mathrm{P}<0.001)$ between the dependent measures for carrying out multivariate analysis of variance. The results of the test of Levene's homogeneity of error variance were not significant $(\mathrm{P}>0.05)$. This suggests that error variance is the same across all levels of group variables.

The results of MANOVA are shown in Table 1. As Table 1 shows, there is a statistically significant difference between two groups in the context of all impulse control sub-tests and general indicator of the scale.

The second hypothesis is that emotional regulation in people with food addiction is different from that of the control group. To investigate this hypothesis, multivariate analysis of variance was used. The results showed that there is a difference between the two groups with regard to at least one variable. The results of the Kolmogorov-Smirnov test was higher than $0.05(\mathrm{P}>0.05)$ in all indices, resulting in data normality. The result of the Bartlett sphericity test was significant $(\mathrm{P}<0.001)$. This indicates that there is a sufficient correlation between the dependent measures for carrying out multivariate analysis of variance. The results of the test of Levene's homogeneity of error variance were not significant $(\mathrm{P}>0.05)$. This suggests that error variance is the same between across levels of group variable. The results of the MANOVA conducted for emotion regulation indices are provided in Table 2. Table 2 shows that there is a statistically significant difference between the two groups in the context of all impulse control sub-tests and general indicator of the scale.

\section{Discussion}

The results showed that there is a statistically significant difference between the food addiction group and the control group in all scale indices of impulse control. This means that food addiction patients have cognitive impulsivity, higher

Table 1. Multivariate analysis of variance for impulse control indicators

\begin{tabular}{ccccccc}
\hline Index & Sum of Squares & $\begin{array}{c}\text { Degree of } \\
\text { Freedom }\end{array}$ & $\begin{array}{c}\text { Mean of } \\
\text { Squares }\end{array}$ & F Ratio & $\begin{array}{c}\text { Significance } \\
\text { Level }\end{array}$ & Size Effect \\
\hline Cognitive impulsivity & 330.20 & 3 & 110.06 & 12.210 & 0.001 & 0.240 \\
Motor impulsivity & 1811.82 & 3 & 603.94 & 27.227 & 0.001 & 0.413 \\
Disorganization & 1925.66 & 3 & 641.88 & 32.081 & 0.001 & 0.453 \\
Total index & 10710.70 & 3 & 357.23 & 35.227 & 0.001 & 0.477 \\
\hline
\end{tabular}

Iranian Journal of
PSYCHIATRY AND CLINICAL PSYCHOLOGY 
Table 2. Multivariate analysis of variance for emotion regulation indices

\begin{tabular}{|c|c|c|c|c|c|c|}
\hline Index & $\begin{array}{l}\text { Sum of } \\
\text { Squares }\end{array}$ & $\begin{array}{l}\text { Degree of } \\
\text { Freedom }\end{array}$ & $\begin{array}{l}\text { Mean of } \\
\text { Squares }\end{array}$ & F Ratio & $\begin{array}{l}\text { Significance } \\
\text { Level }\end{array}$ & Size Effect \\
\hline $\begin{array}{l}\text { Rejection of emotional } \\
\text { responses }\end{array}$ & 339.22 & 3 & 113.075 & 9.521 & 0.001 & 0.198 \\
\hline $\begin{array}{l}\text { Difficulty in purposeful } \\
\text { behavior }\end{array}$ & 639.02 & 3 & 213.008 & 16.293 & 0.001 & 0.296 \\
\hline $\begin{array}{l}\text { Difficulty controlling } \\
\text { impulses }\end{array}$ & 2388.29 & 3 & 796.097 & 51.576 & 0.001 & 0.572 \\
\hline $\begin{array}{c}\text { Lack of emotional aware- } \\
\text { ness }\end{array}$ & 1002.83 & 3 & 334.278 & 20.911 & 0.001 & 0.351 \\
\hline Limited access to strategies & 3038.16 & 3 & 1012.722 & 63.098 & 0.001 & 0.620 \\
\hline Emotional ambiguity & 687.36 & 3 & 229.122 & 18.317 & 0.001 & 0.321 \\
\hline The overall index & 41120.89 & 3 & 13706.964 & 48.963 & 0.001 & 0.559 \\
\hline
\end{tabular}

no-movement, wider no-planning and weaker performance on impulse control compared to the control group.

The findings of this study are consistent with many other findings regarding drug-dependent individuals, where impulsivity plays a role as the background and the risk factors in the such disorder (drug dependency and also all of the behavioral addiction) [49-57] Also, in studies regarding overeating and food addicts in which impulsivity, again, plays the role of facilitator and risk factor, similar results were obtained $[58,59]$. Diret al. (2013) found evidence showing that drug and alcohol use as well as frequent overeating and its subsequent clearance are impulsive triggers [60]. The core (center) impulsivity is negative emotions in which the person does an impulsive act while experiencing the negative emotions simultaneously. So, impulsive actions are attempts to regulate negative emotions [61].

In fact, the results of different studies have shown that emotion regulation is not only one of the most important and influential causes but also the maintaining factor of drug dependence disorder [63]. Drug dependence and continued use, in fact, is the result of the low level of emotional regulation strategies and the inability to deal effectively with emotions and manage them, especially in cases of first-time drug use. On the other hand, it seems that the same process occurs in people with food addictions as well. The results obtained in this study are consistent with the research conducted by Levitan and Davis (2010), Desmetand Schifferstein (2008), Dryanz et al (2011), Macht and Simon (2000), Brown et al (2009), Macht (2008), Gearhardt et al (2012) and Baldovsky et al. (2015) in which emotion dysregulation led to eating disorders and pathological overeating [39-41, 64-67]. In fact, according to the results of this study and other studies, it seems that people with food addiction overeat and consume their food as a defense mechanism in the face of negative emotions and low self-esteem. This mechanism is similar to drug addiction, which highlights the role of depression, anxiety, negative mood and lack of emotional regulation as incentives in drug use or food intake [8]. Generally, the results of this study are consistent with the results of Khantzian's self-treatment theory. From this perspective, addictive behaviors are rooted in intolerable and painful emotions such as depression, anxiety, shame, anger and sense of helplessness, and drug use is an attempt to compensate for the shortcomings of the ego in defense against these emotions. This view is in line with the frequency of clinical observations and studies emphasizing the important role of human psychological suffering in addictive disorders [63]. According to it, treatment for these patients consists of conscious methods of controlling impulses and desires as well as effective methods of creating emotion regulations [65].

\section{Acknowledgments}

This research was extracted from the MSc. thesis of the first author, in the Department of Clinical Psychology, Shool of Behavioral Sciences and Mental Health (Tehran Institute of Psychiatry), Iran University of Medical Sciences, Tehran, Iran.

\section{Conflict of Interest}

The authors declared no conflicts of interest. 


\title{
مقايسه كنترل تكانه و نظمبخشى هيجانى در بيمار ان مبتلاو غير مبتلا به اعتياد غذايى
}

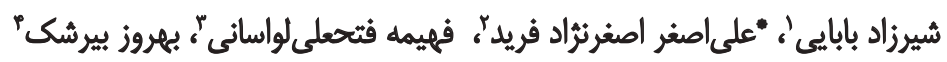

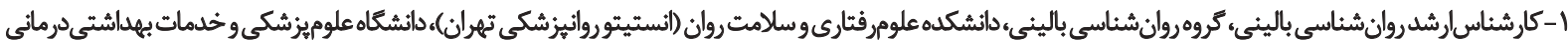
ايران، تهران، ايران.

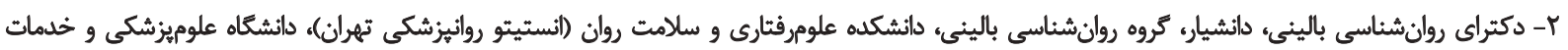

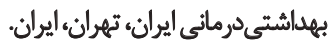

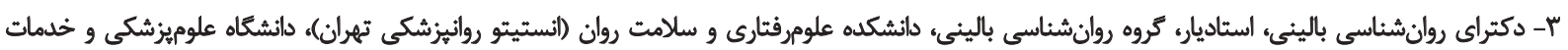
بهداشتى درمانى ايران، تبهران، ايران.

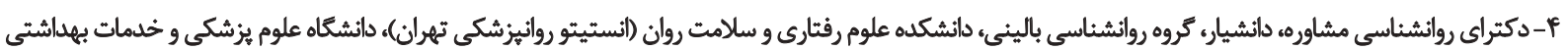
درمانى ايران، تهران، ايران.
\end{abstract}

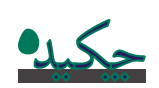

هداف هدف يُروهش حاضر، بررسى متغيرهاي كنترل تكانه ونظمبخشى هيجانى در بيماران مبتلابه اعتياد غذايى درمقايسهبا كروه كنترل بود.

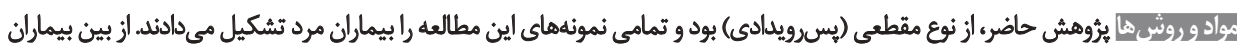

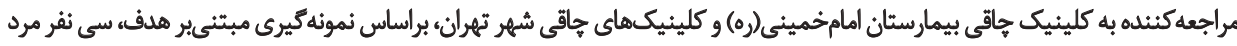

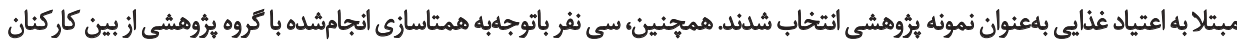

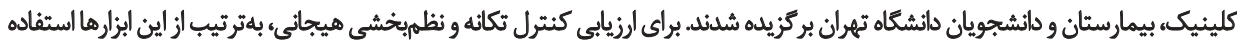

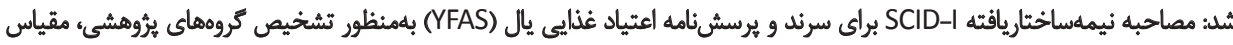

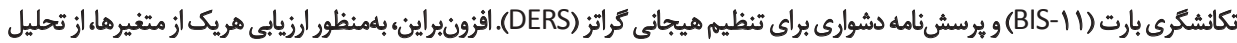

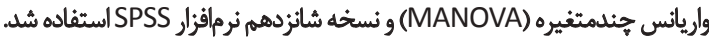

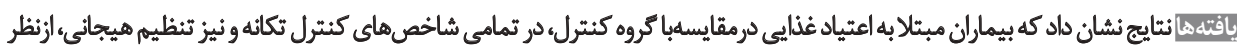
آهارى نمره كمترى كسب كريند.

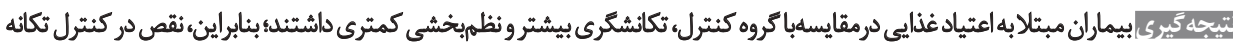

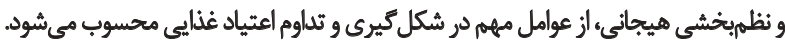

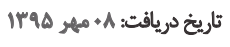

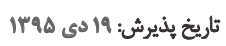

: كإن اعتياد غذايي، كنترل ثكانه. نظمبخشى هيجانى

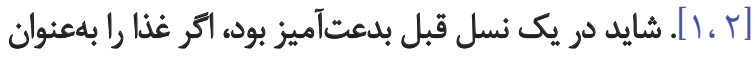

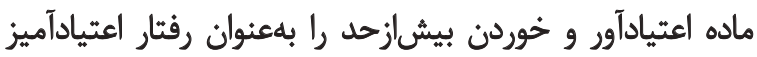

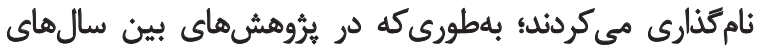

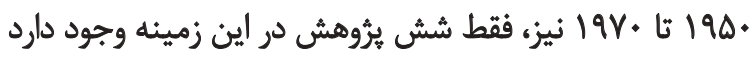

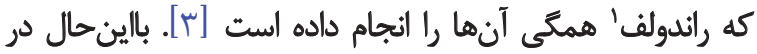

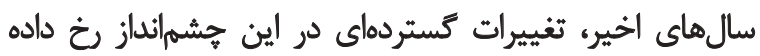

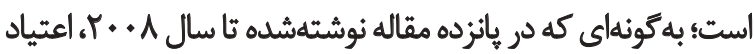

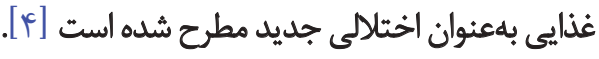

1. Randolf
اعتياد، اختّلال مزمن اكتسابى عوديذير است كه با برخى

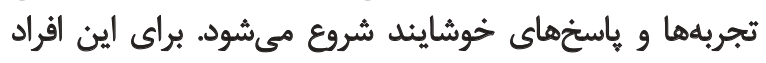

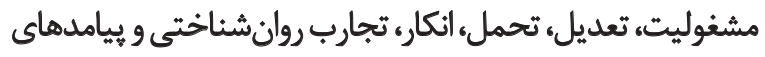

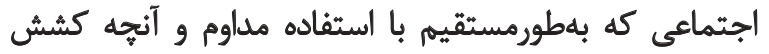
مخرب بين مواد (فعاليتى مائد قماريازى) و بيماران ناميده

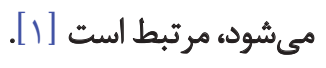
در ميان مردم و متخصصان، اين عقيدهاى بذيرفنتى است كه ونه

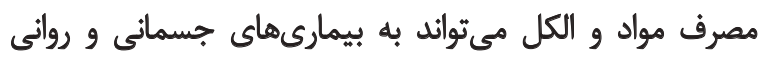
كوناكونى منجر شود. امروزه، متخصصان اين حوزه معتقدند قمار و و

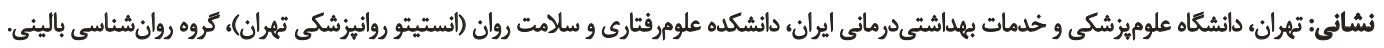
تلفن:

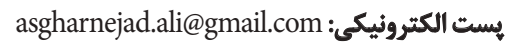




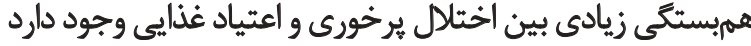

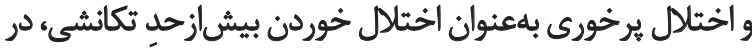

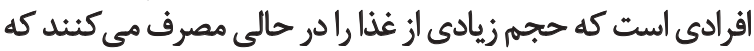

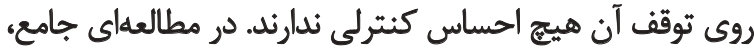

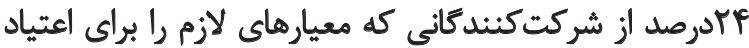

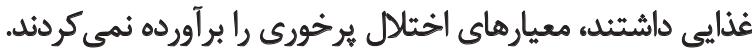

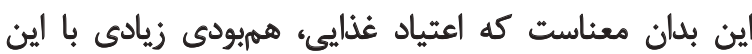

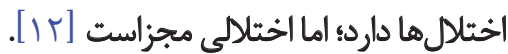

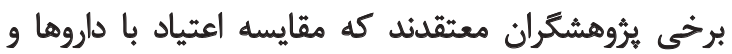

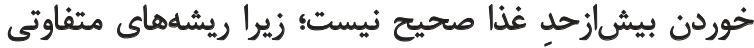

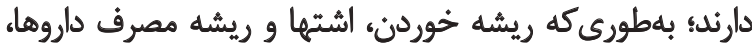

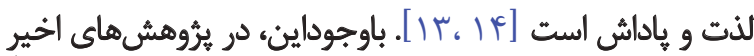
انجامشده روى موشها، نشان داده شده كه مسيرهاى عصبى لئى

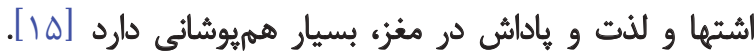

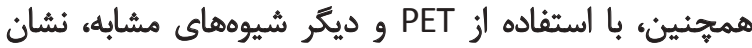

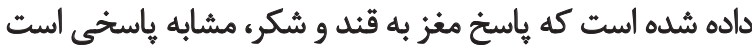

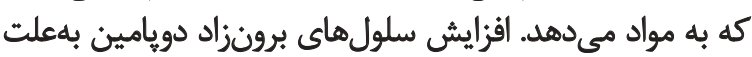

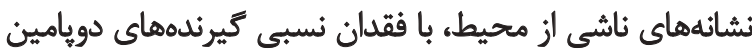

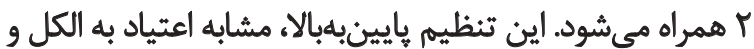

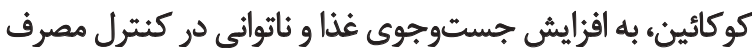

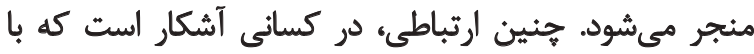

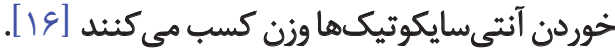

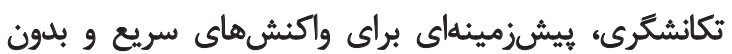

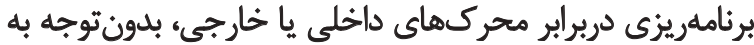

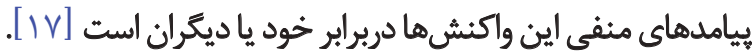

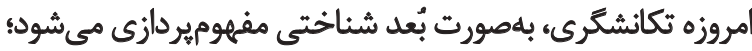

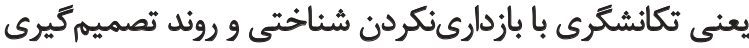

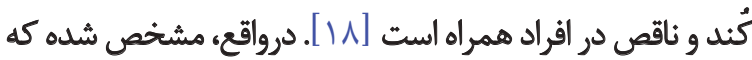

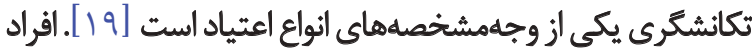

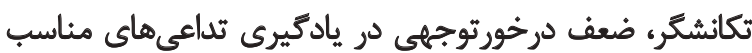
بين ياداش و تنبيه دارند كه براى انتخاب مناسب، از از اهميت

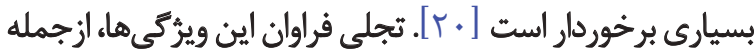

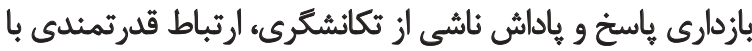

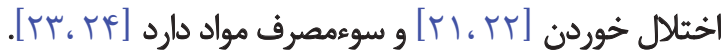

مرور مطالعات انجامشده در حوزه تكانشُّرى نشان مىدهد

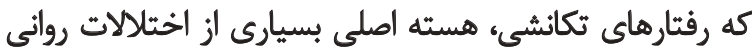

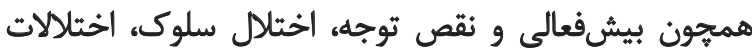

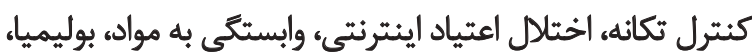

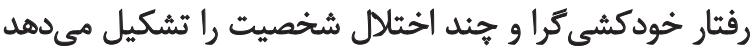

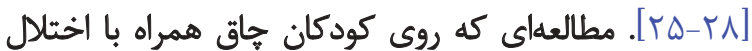
خوردن و بدون آن انجام شده نشان داد كه بيماران بان بان اختلال
كير هارت و و همكاران، به عناصر مرتبطبا وابستَكى به مواد اشاره

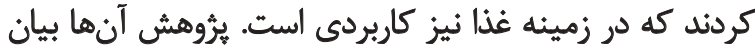

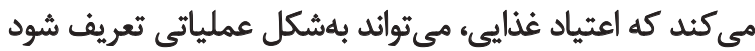

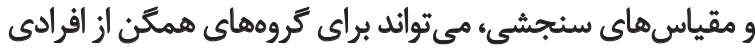

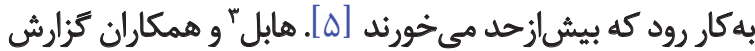

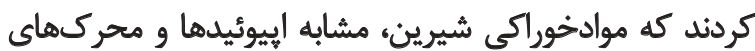

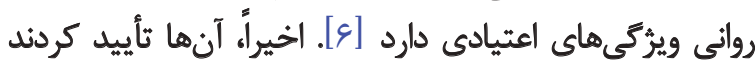

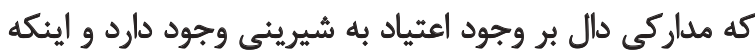

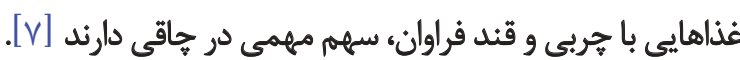
نشانههايى كه مى توان به اعتياد غذايى نسبت داد، عبارت است ازئ

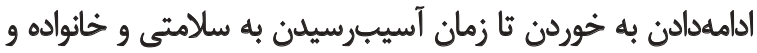

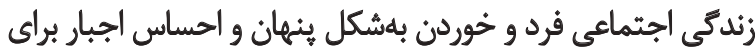

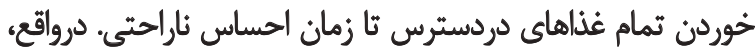

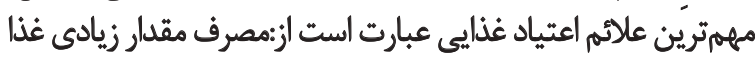

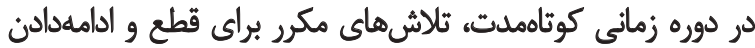

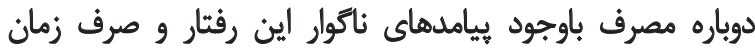

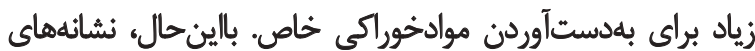

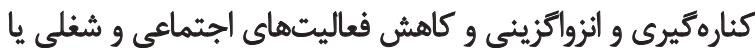

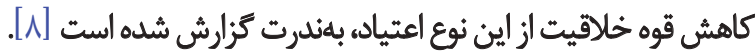
با استفاده از مقياس YFAS" نشان داده شده كه در ميان

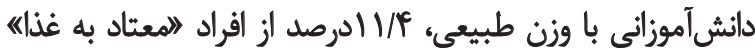

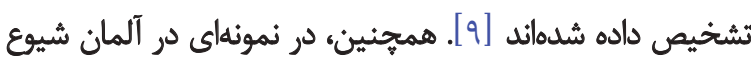

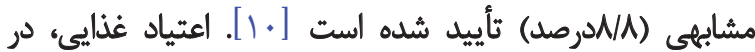
راهنماي تشخيصى و آمارى اختلالات روانى (DSM-IV-TR) طبقهبندى خاصى ندارد؛ ولى با بسيارى از اختلايلات ائن إين مجموعه

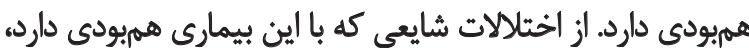

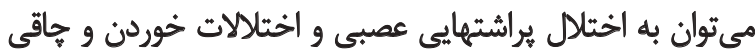

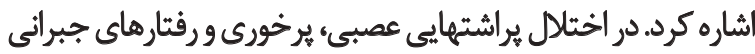

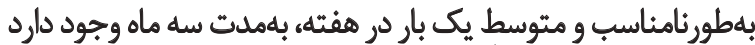

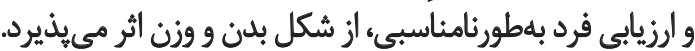

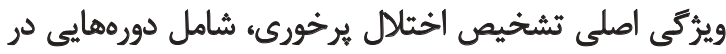

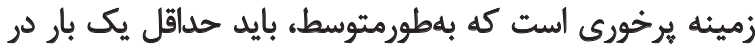

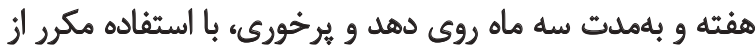

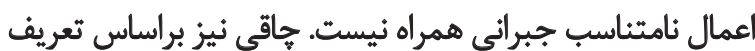

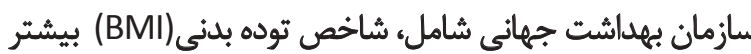

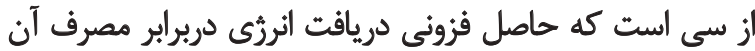

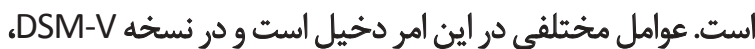

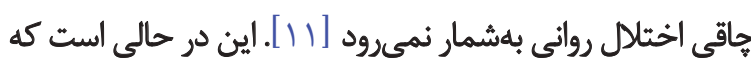

2. Gearhardt

3. Hoebel

4. Yale Food Addiction Test (YFAT) 
هيجانها نقش مهمي در انتخاب غذا و رفتارهاي خوردن دارد

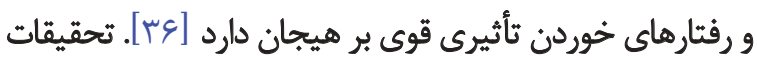

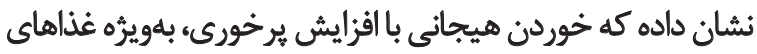

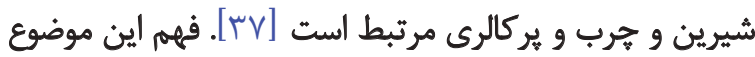

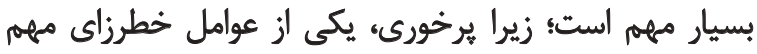

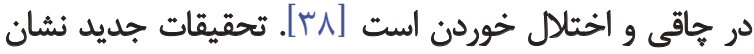

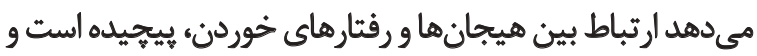

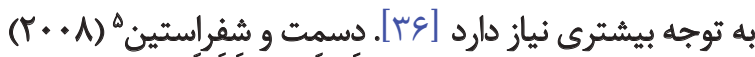

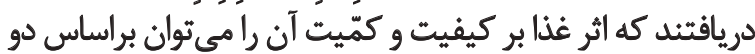

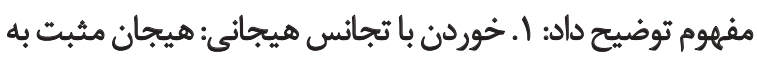

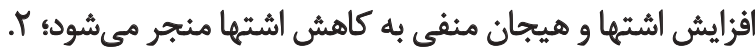

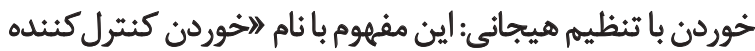

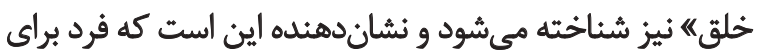

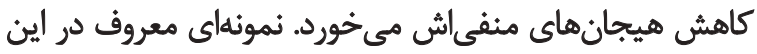

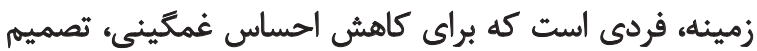

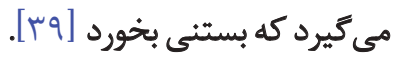

براساس ديدكاه آدريانز و ريدر و ايورز (11 (Y)، مفهوم خوردن

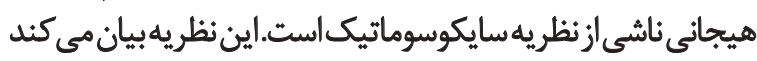

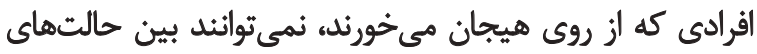

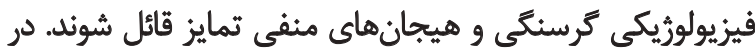

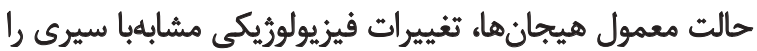

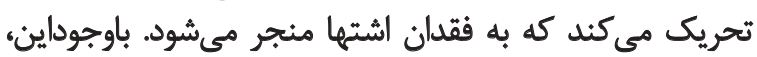

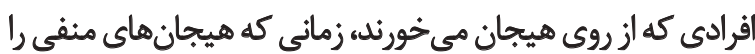

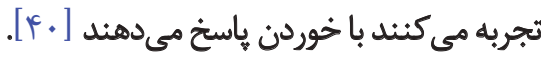

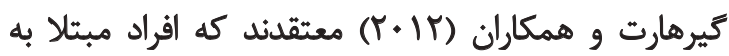

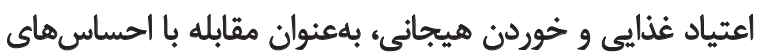

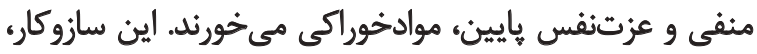

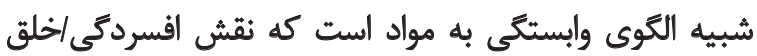

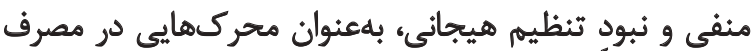

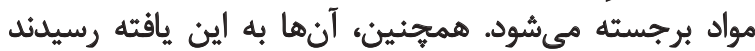

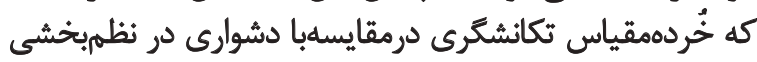

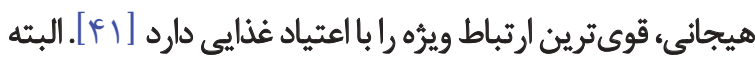

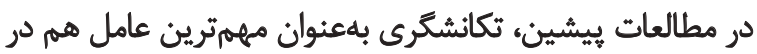

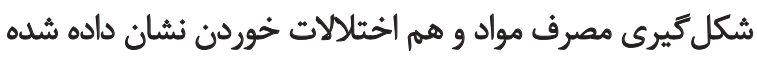

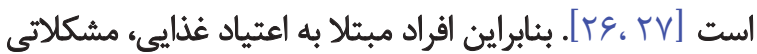

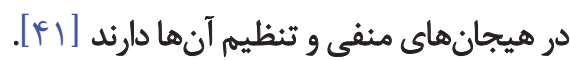

همانطوركه بيان شده يكى از علل ايجادكنيده و تداومبخش

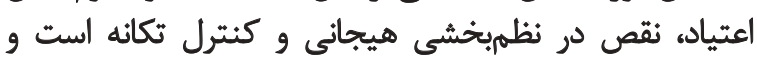

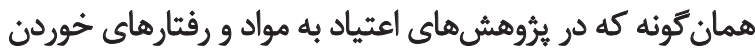

5. Desmet \& Schifferstein

6. Adriaanse, Ridder, \& Evers

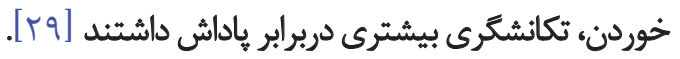

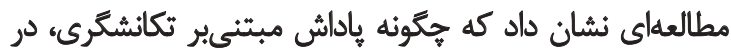

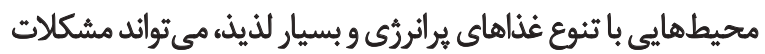

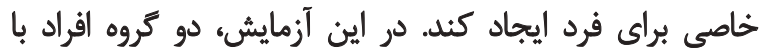

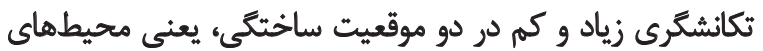

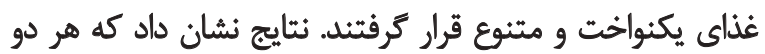

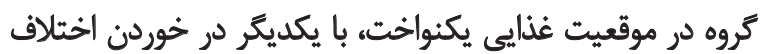

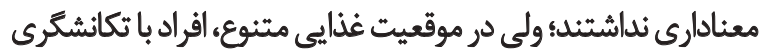

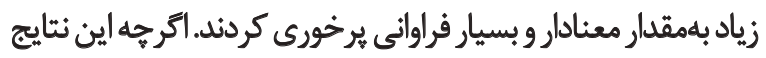

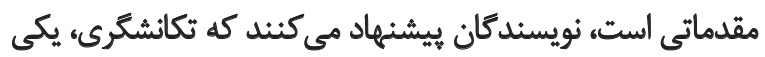

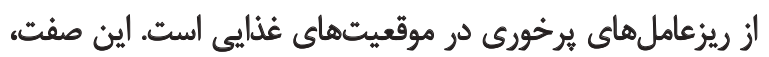

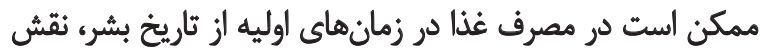

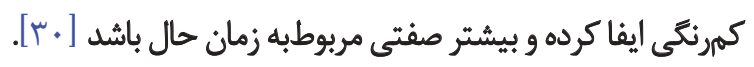
در ديدكاه تكاملى، هيجان ميراثى است كه از انسانهاي

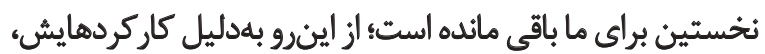

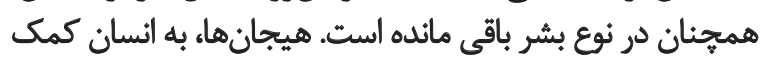

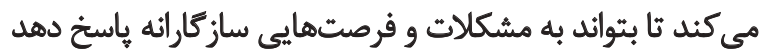

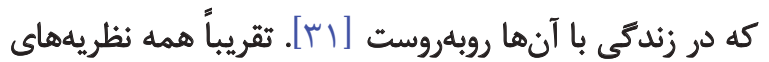

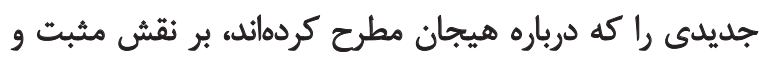

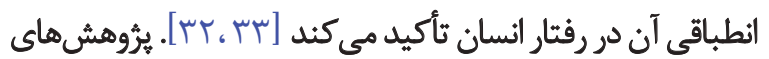

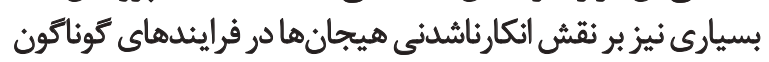

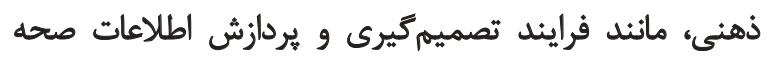

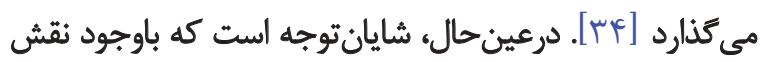

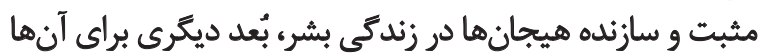

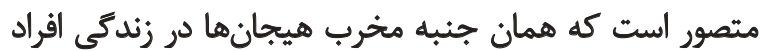

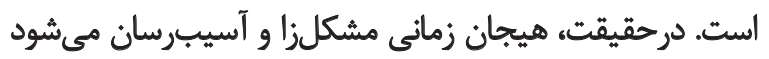

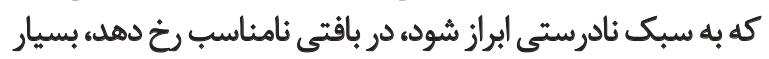

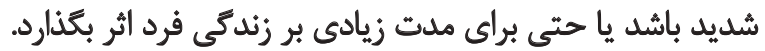

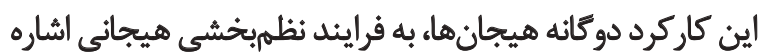

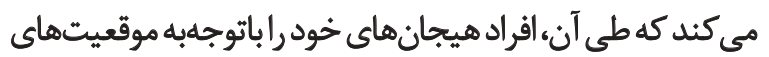
كوناكون، تنظيم و تعديل مى كنيند [هب]. نظمهبخشى هيجانى رافرايندهاى درونى و بيرونى مسئول يايش

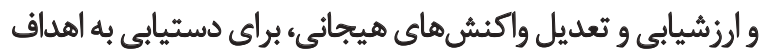

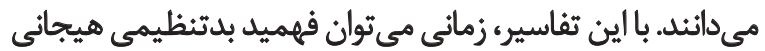

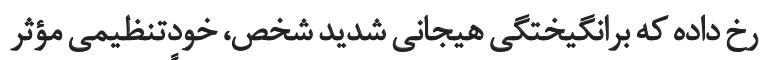

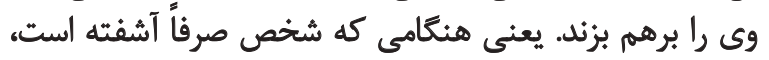

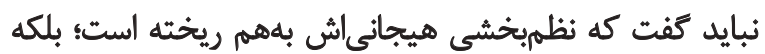

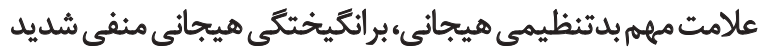

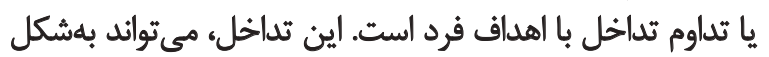

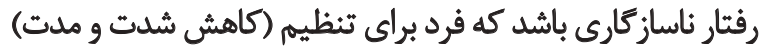

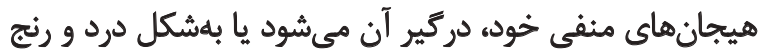

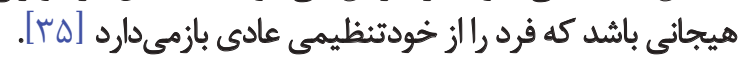




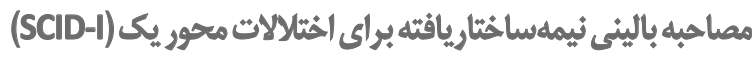
اين مصاحبه بالينى، ابزار جامع استانداردشدهاي براي ارزيابى

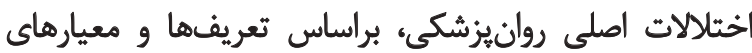

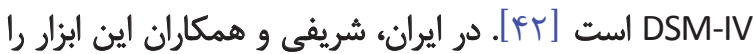

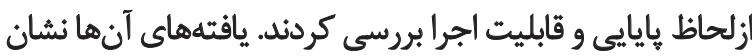

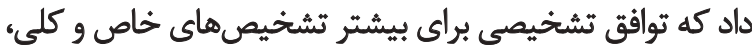

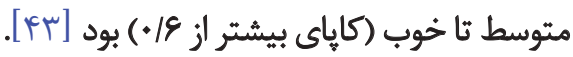

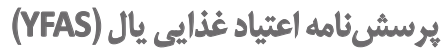

مقياس اعتياد غذايى يال، براى تشخيص و شناسايى افرادى

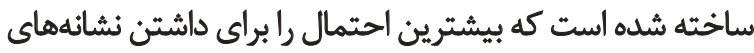

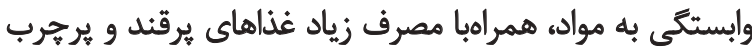

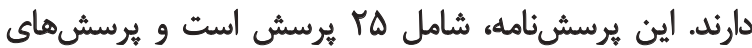

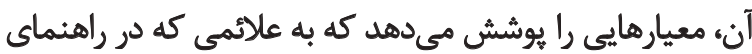

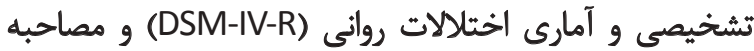
بالينى ساختاريافته براى اختلالات DSM، بامنظور تشخيص

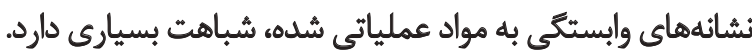

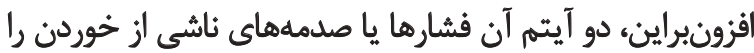

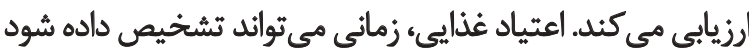

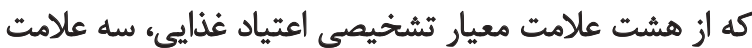

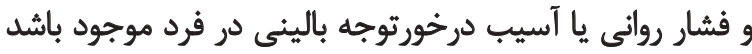

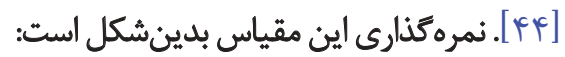
نقطهبرشهاى زير، براى يرسشهاي مستمر طراحى شده است.

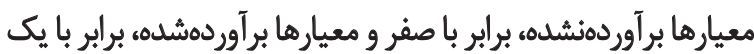

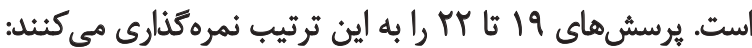
صفر=(·) و يك=(1)؛

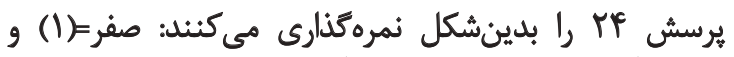

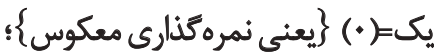

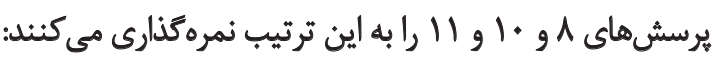

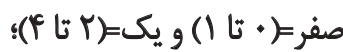

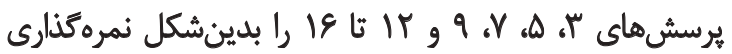

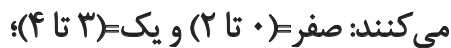
هرسشهاى ا، Y، f و و و را بدينترتيب نمرهكذارى مي كنيند:

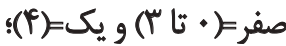

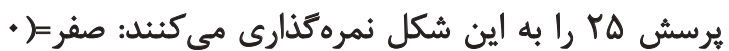
تأf)

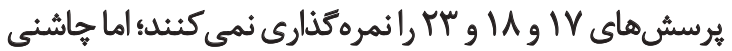

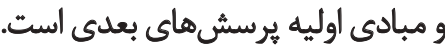

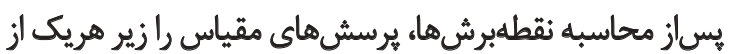

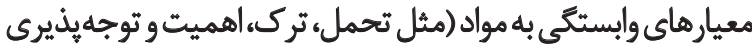

و جاقى آورده شده، به اهميت نقش اين مؤلفهها در زمينه

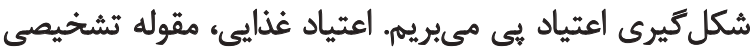

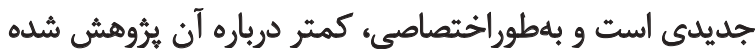

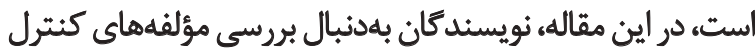

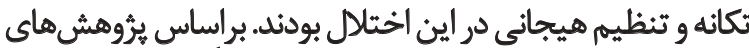

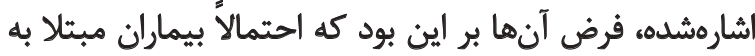

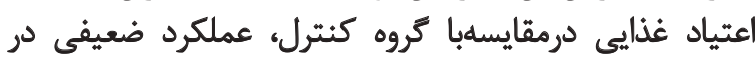
مؤلفهاى كنترل تكانه و تنظيم هيجانى دارند.

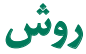

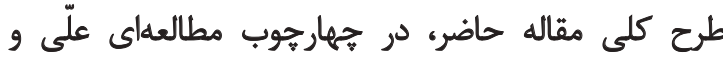

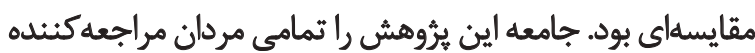

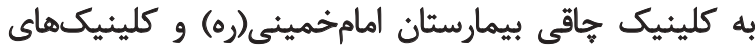

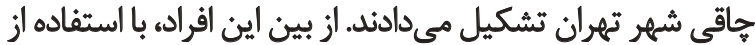

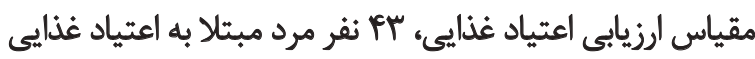

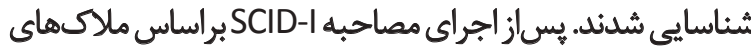

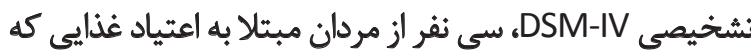

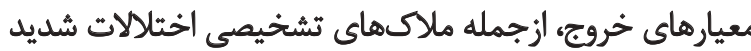

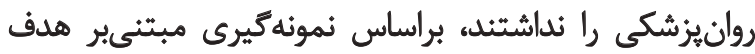

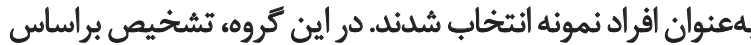

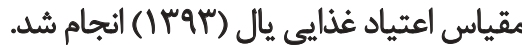

كروه كنترل را افرادى دربرمى كرفت كه به اعتياد غذايى مبتلا

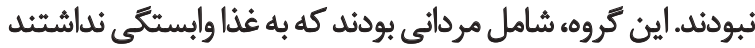

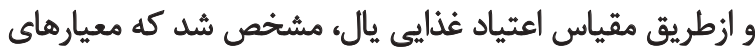

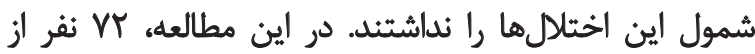

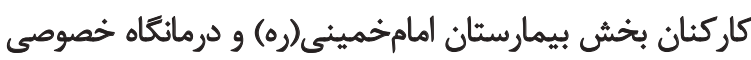

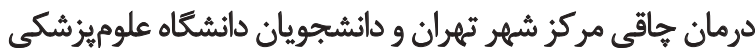

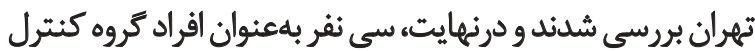

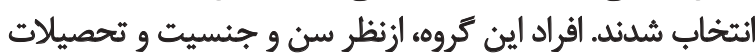

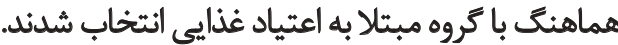

ملاكهاي ورود افراد به اين يُروهش عبارت بود ازئ رضايت

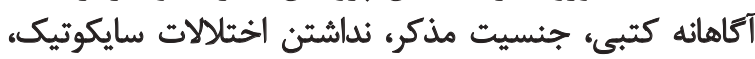

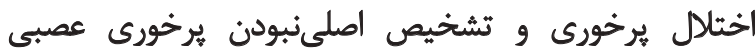

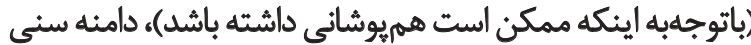

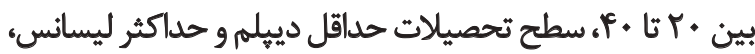

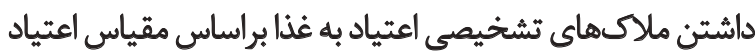

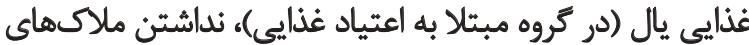

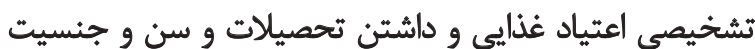
هماهنگ با كروه مبتلا به اعتياد غذايى (در گروه كنترل). ابزارهاى مهم اين ثروهش، عبارت بود از: 
ازطريق محاسبه آلفاى كرونباخ بهدست آمد،

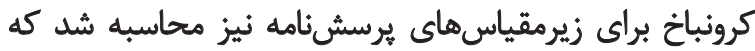

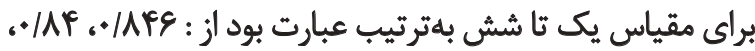
به

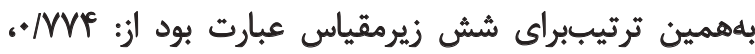


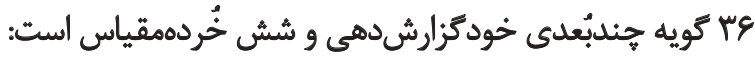

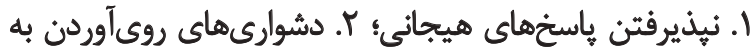

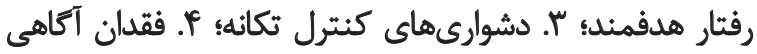

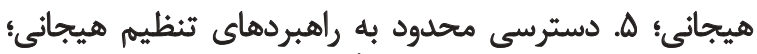

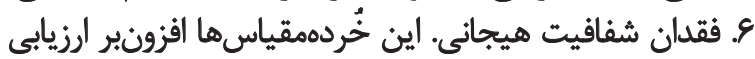

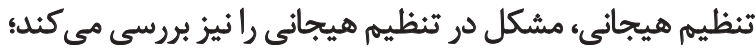

$$
\text { اما بيشتر بر مشكلات متمركز است [FV] }
$$

براي تجزيهوتحليل دادههاي بهدستآمده، از نسخه شانزدهمم

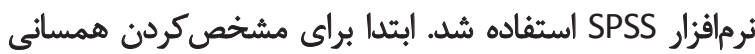

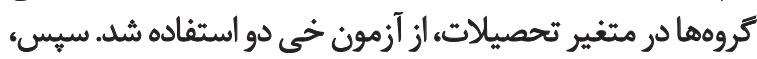

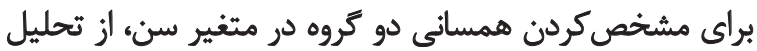

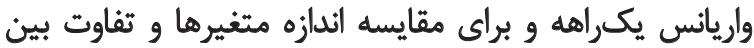

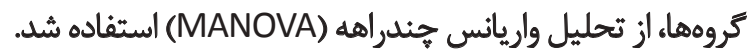

يافتهها

درمجموع، شصت نفر (سى نفر در تروه مبتلا به اعتياد غذايى

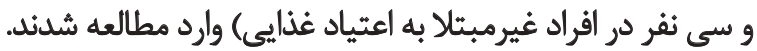

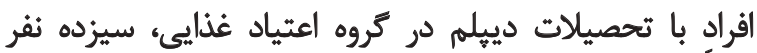

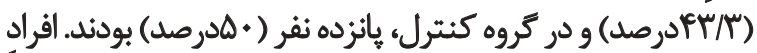

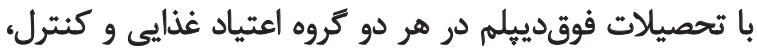
هشت نفر (VIN

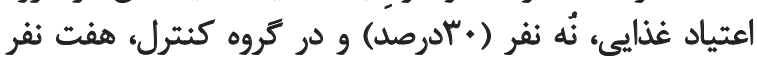

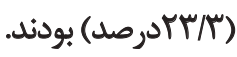

افزونبر جنسيت كه فقط شامل آزمودني هاى مرد ميشيد،

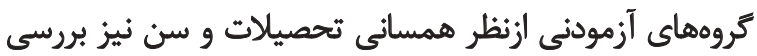

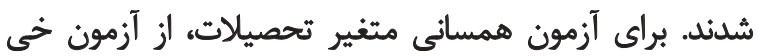

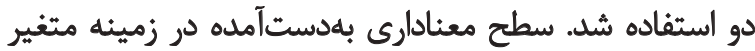

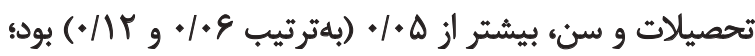

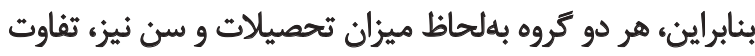

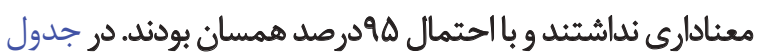

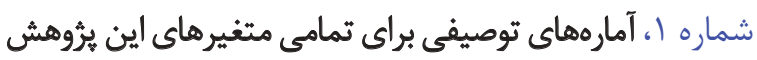

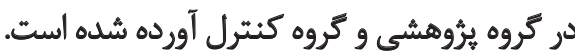

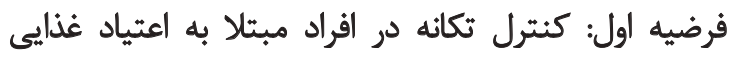

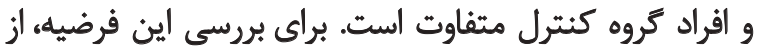

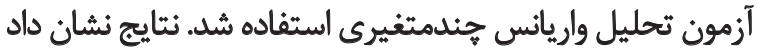

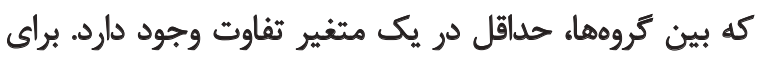

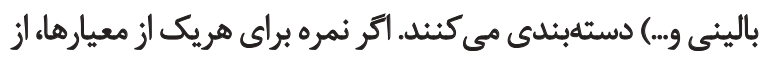

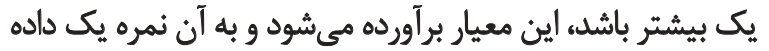
مىشود. اكر نمره صفر شود، آن معيار برآورده نمى ئشود.

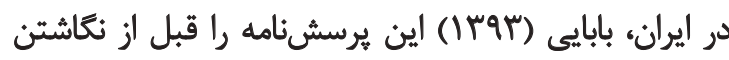

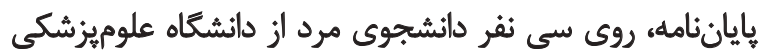

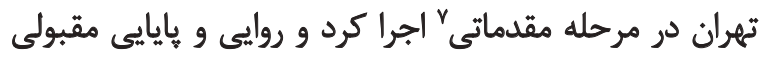

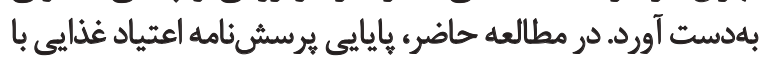

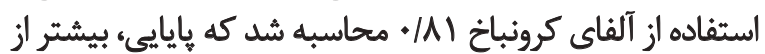

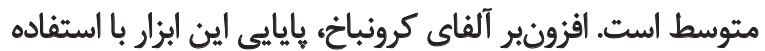

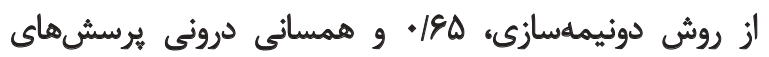

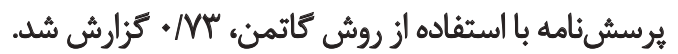

مقياس نكائشكرى بارت (BIS-11)

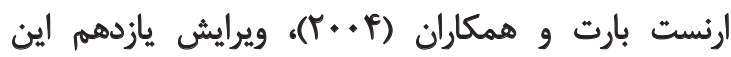

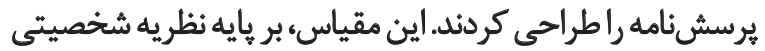

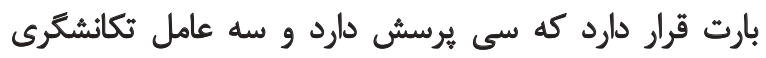

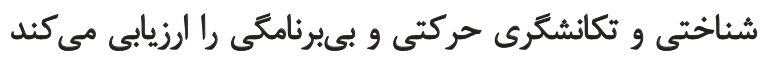

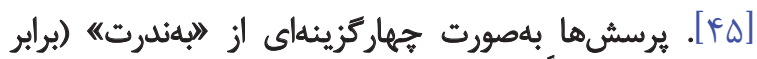

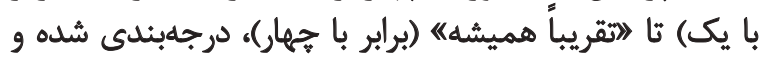

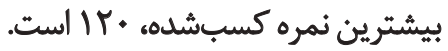

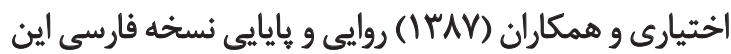

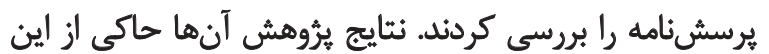

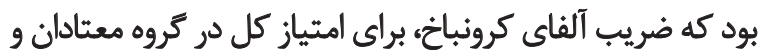

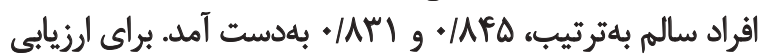

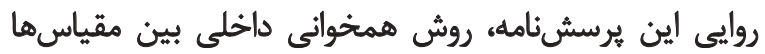

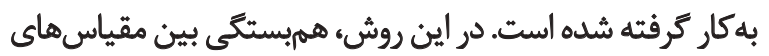

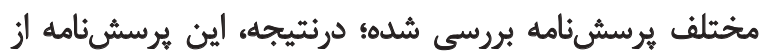

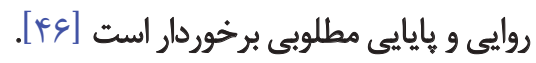

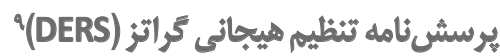

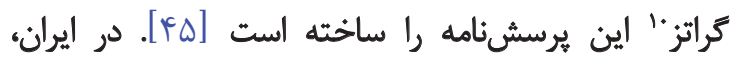

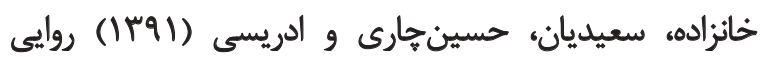

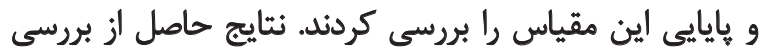

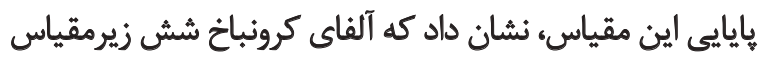

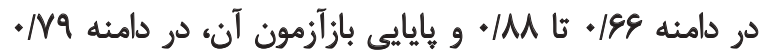

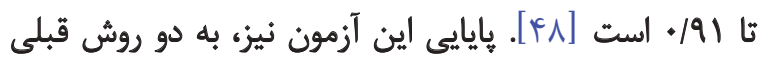

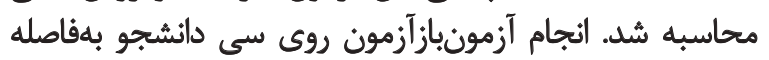

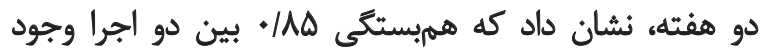

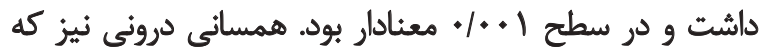

7. Pilot

8. Barratt Impulsiveness Scale (BIS)

9. Difficulties in Emotion Regulation Scale (DERS)

10. Gratz 
جدول ا. ميانكين و انحراف استائدارد شاخص هاى مربوط به تكانشكّى و دشوارى در نظمبخشى هيجانى

\begin{tabular}{|c|c|c|}
\hline مياتئين (انحراف اسثاندارد) & نوع كروه & شاخص هاى تكائشكّرى \\
\hline $\begin{array}{l}\text { WIY(Y/FE) } \\
\text { IF/IE(Y/MU) }\end{array}$ & اعتياد غثلايي & تكانشكرى شُناختمى \\
\hline $\begin{array}{l}T V / E \cdot(T / M I) \\
I V / T T(Y / \cdot T)\end{array}$ & أعتياد غُذايِي & تكانشكرى حركتى \\
\hline $\begin{array}{l}r V / A T(\varphi / I V) \\
W \cdot g(\varphi / R T)\end{array}$ & اعتياد غُذايب & بعبرنامكىى \\
\hline 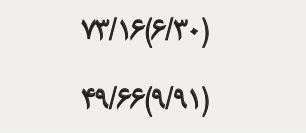 & اعتياد غذايي & شاخص كلى \\
\hline & موارى در نظمج & \\
\hline $\begin{array}{l}1 f / N \cdot(r / N+) \\
1+/ r g(Y / H))\end{array}$ & اعتياد غذايبى & ئيذيرفن هاسخهاى هيجانى \\
\hline $\begin{array}{l}W \cdot g(T / Y \Lambda) \\
W / \varepsilon \cdot(Y / \cdot M)\end{array}$ & 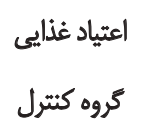 & دشوارى در رفتار هدفمند \\
\hline $\begin{array}{l}19 / 94(\% / \cdot 1) \\
9 / 9 \cdot(\% / / 1)\end{array}$ & أعتياد غذذايي & دشوارى كنترل تكانه \\
\hline $\begin{array}{l}r \cdot / E \cdot(\varphi / M)) \\
\mid M / \varepsilon r(\varphi / \cdot \lambda)\end{array}$ & اعتياد غذأيى & فقدان أكاهى هيجانى \\
\hline $\begin{array}{l}r \& / \Lambda \cdot(1 / N \Lambda) \\
\mid r / \Delta \&(Y / \cdot V)\end{array}$ & اعتياد غذأيى & دسترسي هحدود به راهبردها \\
\hline $\begin{array}{l}I r / V \&\left(Y / q_{+}\right) \\
N T \cdot(Y / q \Delta)\end{array}$ & اعتياد غَذليى & إيهام هيجائى \\
\hline 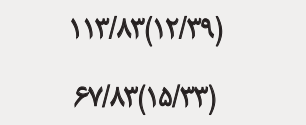 & اعتياد عُدَايي & شاخص كلى \\
\hline
\end{tabular}

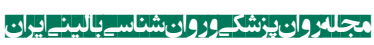

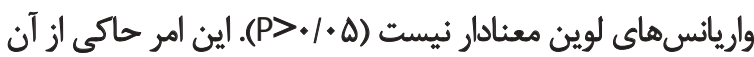

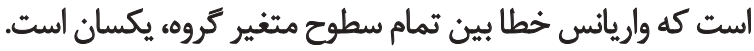
براى ارزيابى هريك از شاخص هاى كنترل تكانه، تحليل واريانس

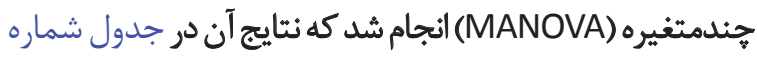

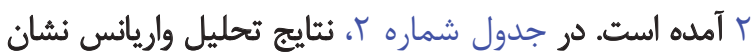

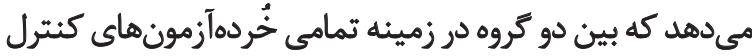

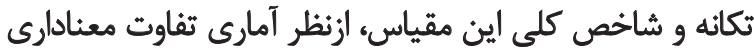

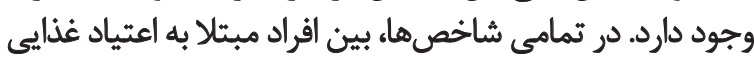

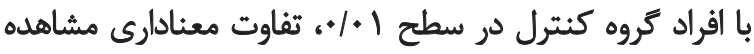

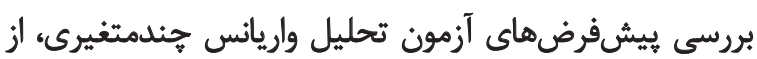

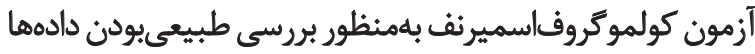

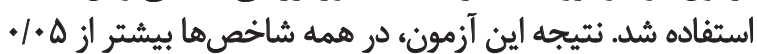

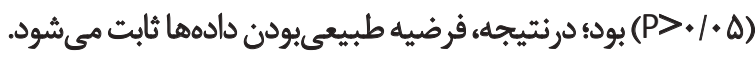

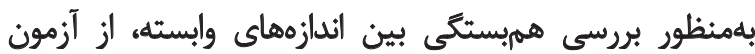

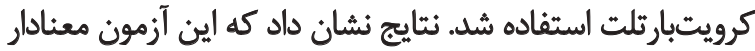

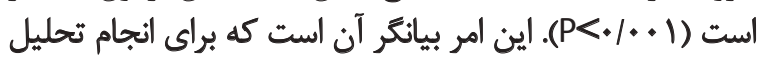

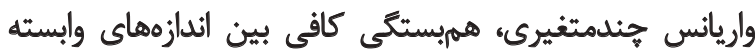

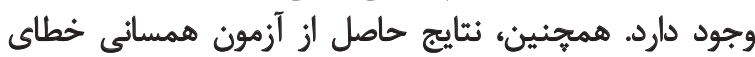


جدول r. تحليل واريانس جندمتغيره شاخصهاي كنترل تكائه

\begin{tabular}{|c|c|c|c|c|c|c|}
\hline اندازه اثر & سطحمعادارى & نسبت F & ميانكين مجذورات & دوجه أزادى & مجموع مجذورات & شاخص \\
\hline.$/ M P$ & $.1 . .1$ & $|r / T|$. & 11.1 .8 & r & $m \cdot / r$ & تكائشكرى شُناختي \\
\hline . pir & $.1 . .1$ & TVRTY & E.r/qr & $r$ & MII/AY & تكانشكرى حركتى \\
\hline . pear & $.1 . .1$ & $M / \cdot A 1$ & giv/M & $r$ & $1950 / 88$ & بي برنامكى \\
\hline.$/ F W$ & $.1+.1$ & TA/TTY & rQV+/r & $r$ & $1+V 1 \cdot N$ & شاخص كلى \\
\hline
\end{tabular}

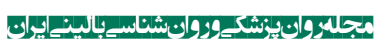

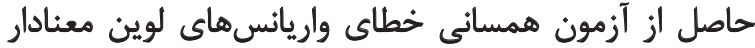

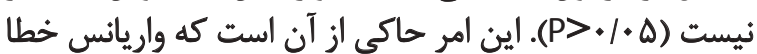
بين تمام سطوح متغير كروه، يكسان است.

براى ارزيابى هريك از شاخصهاى نظمبخشى هيجانى، تحليل واريانس خندمتغيره (MANOVA) انجام شد كه نتايج

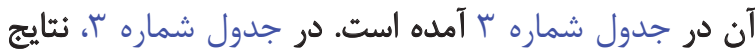

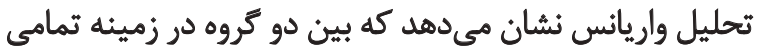

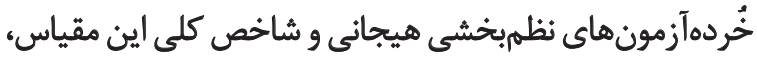

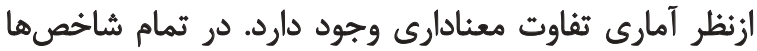

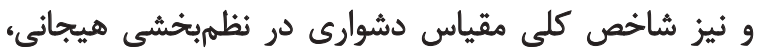

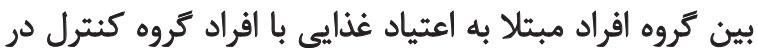

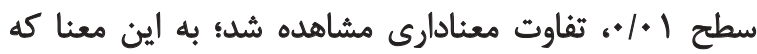

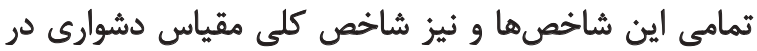

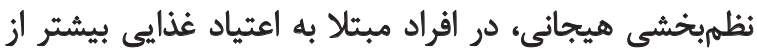

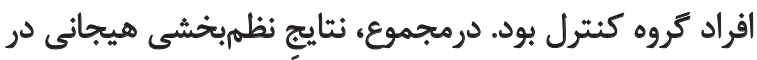

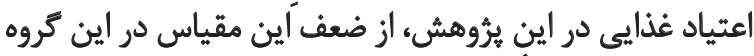

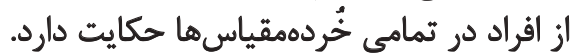

بحث

نتايج نشان مي دهد كه بين افراد مبتّلا به اعتياد غذايى با افراد
شد؛ اين يافته بدينمعناست كه تمام زيرمقياس تهاى مقياس

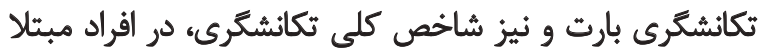

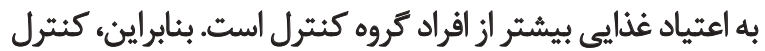

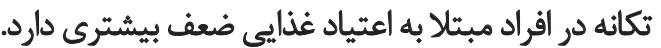
فرضيه دوم: نظمبخشى هيجانى در افراد مبتلا به اعتياد

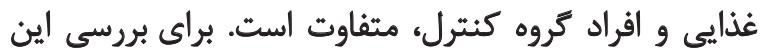

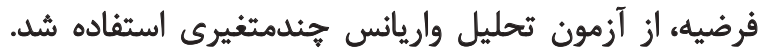

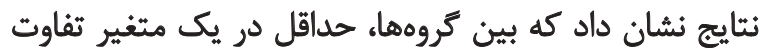

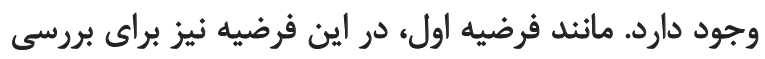

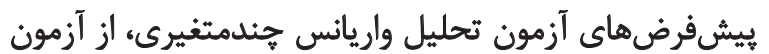

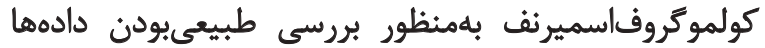

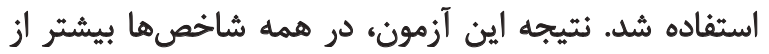

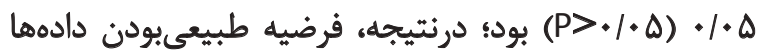

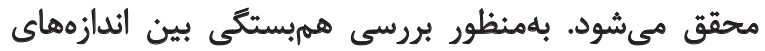

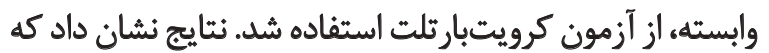

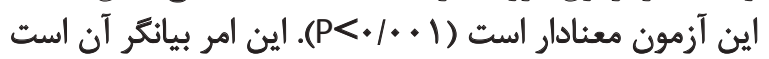

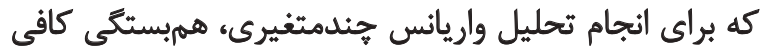

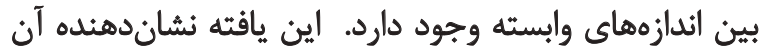

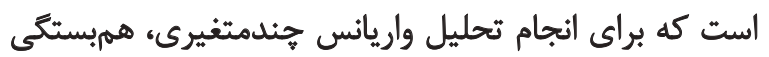

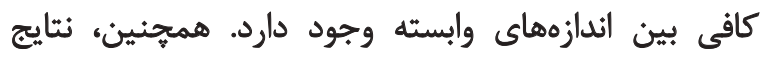

جدول ז. تحليل واريانس جندمتغيره شاخصهاى نظمبخشى هيجانى

\begin{tabular}{|c|c|c|c|c|c|c|}
\hline اندازه اثر & سطح معنادارى & نسبت F & ميانتين مجذورات & درجه آزادى & مجموع مجذورات & شاخص \\
\hline.$/ 14$ & $.1+.1$ & VATI & $11 \% / \cdot V \Delta$ & $r$ & $M \pi / r r$ & ثيذيرفتن ياسخخاي هيجانى \\
\hline.$/ T q$ & $+* 1$ & $18 /$ rar & $M I r / \cdots A$ & $r$ & $q r q+r$ & دشوارى در رفتار هدفمند \\
\hline$\cdot / \Delta V$ & $.1 . .1$ & DY /OVE & V981.9V & $r$ & TrNTI & دشوارى كثترل تكائه \\
\hline.$/ T \Delta 1$ & $.1 . .1$ & $r+1911$ & $M e / M r A$ & r & $1 . . r / A r$ & فقدان آكاهي هيجاني \\
\hline . /ET. & $.1 .+1$ & $8 \pi / \cdot 21$ & 1.IT/MT & r & r.rNIE & دسترسى محلدود به راهبردها \\
\hline.$/ M T I$ & $\% 1$ & WMIV & Mrg/IrT & r & EAV/TE & ابهام هيجائي \\
\hline$\cdot / \Delta \Delta q$ & $+1+\infty$ & PNQGT & ItV+g/agt & $r$ & FIIT-119 & شاخص كلى \\
\hline
\end{tabular}

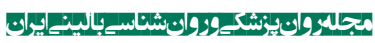




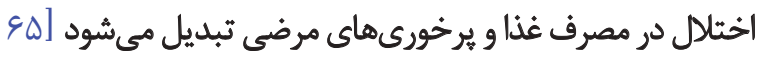

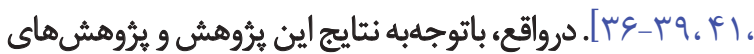

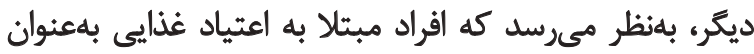

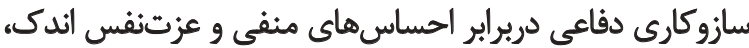

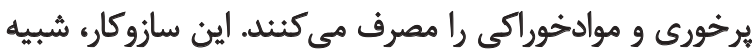

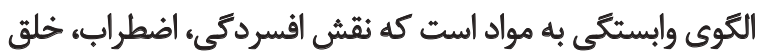

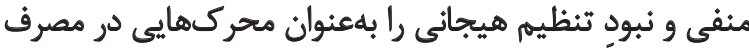

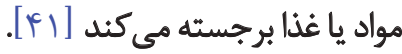

درحقيقت افرادمبتلابهاعتيادغذايى،درواكنش بهاسترسورهاى بإن

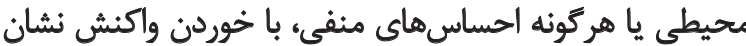

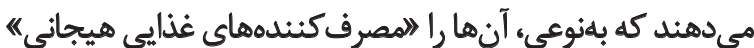

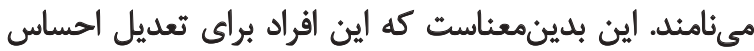

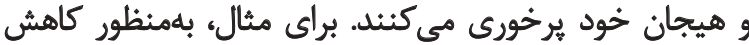

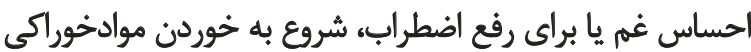

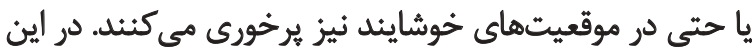

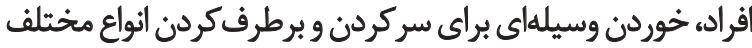

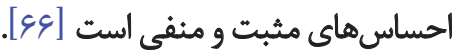

\section{تتيجلمكيرى}

همانطور كه در مقدمه اشاره شد، نقش كنترل تكانه و

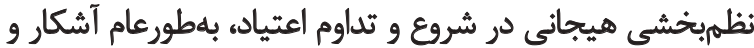

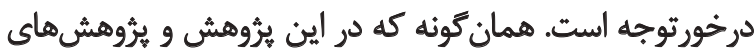

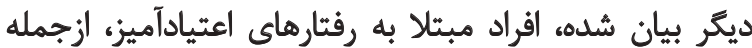

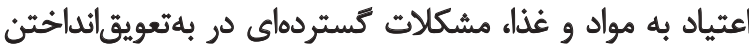

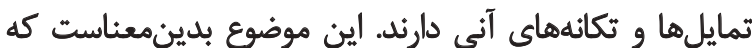

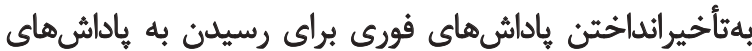

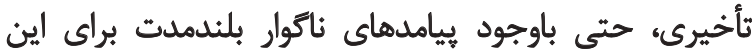

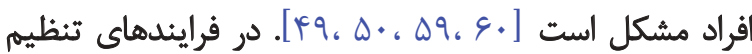

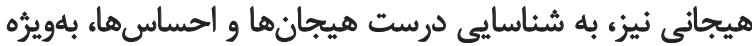
احساسهاي منفى و استفاده از راهبردهاي مناسب براني براي ياستخ

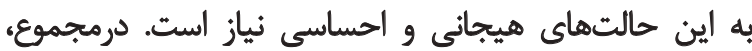

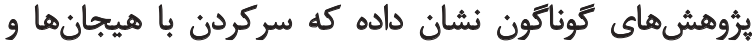

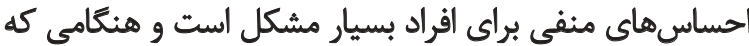

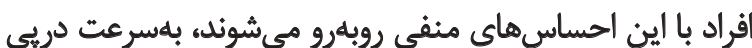

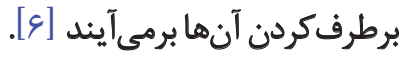

يُروهش ها در حوزه اعتياد نشان داده كه افراد مبثلا به اعتياد،

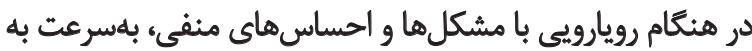

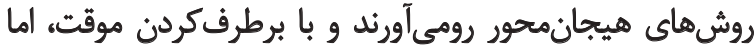

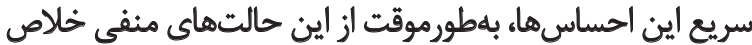

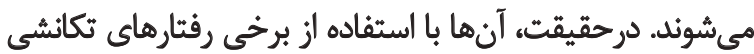

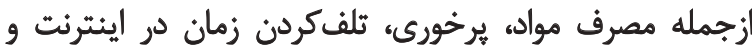

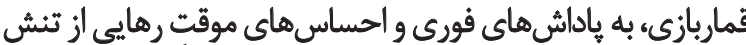

كروه كنترل، در همه شاخصهاى مقياس كنترل تكائه تفاوت

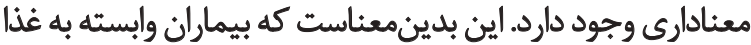

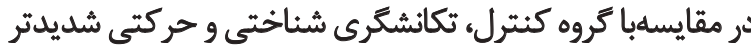

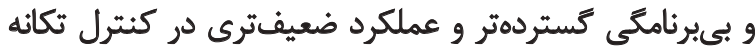

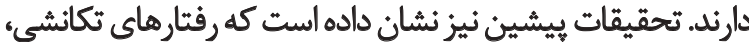

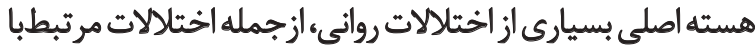

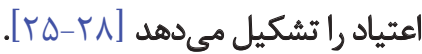

يافتهاى اين مطالعه، با بسيارى از يافتههاى مطالعات

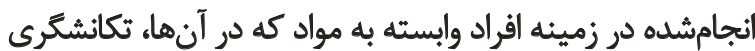

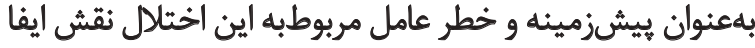

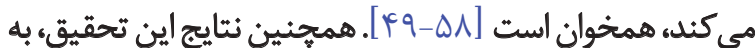

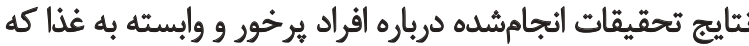

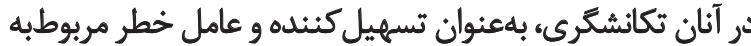

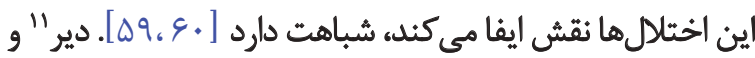

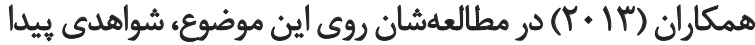

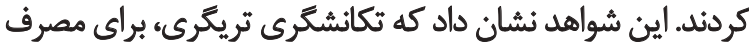

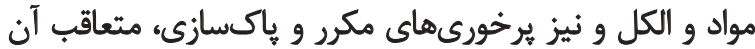

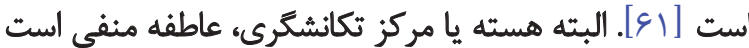

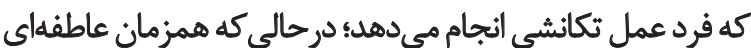

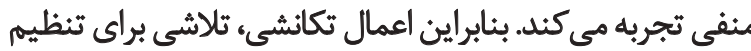

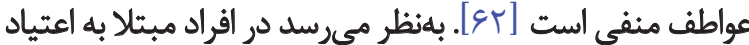
غذايى، جنين موضوعى اتفاق مى افتتد.

بين افراد مبتلا به اعتياد غذايي با افراد گروه كنترل، در همه

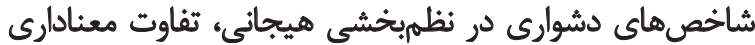

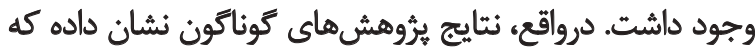

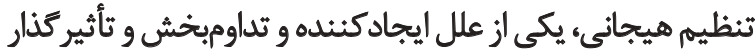

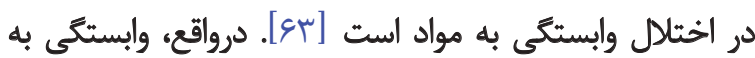

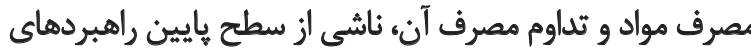

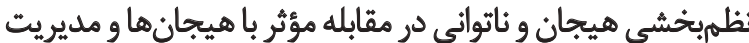

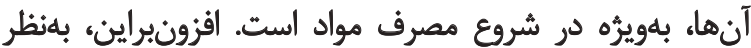

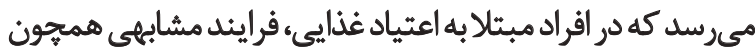

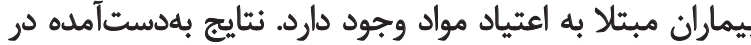

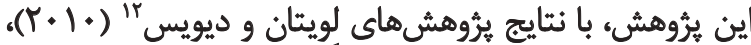

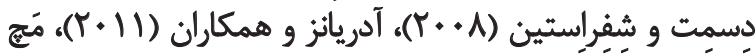

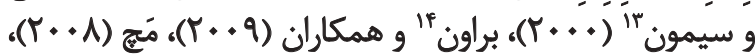

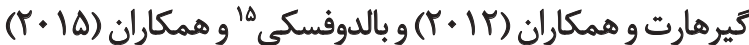

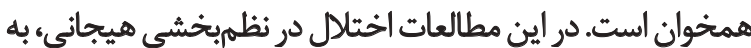

11. Dir

12. Levitan \& Davis

13. Macht \& Simons

14. Brown

15. Baldovsky 


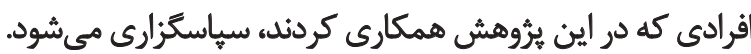

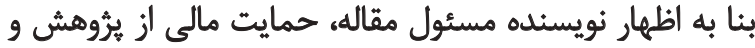
تعارض منافع وجود نداشته است.
هميرسند. با اينكه اين رفتارها عواقب بلندمدت تُستردهاي دارد؛ اما

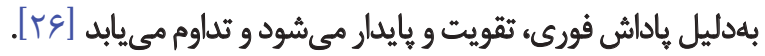
بلطوركلى، نتايج اين ثرؤهش با نتايج نظريه خوددرمانى

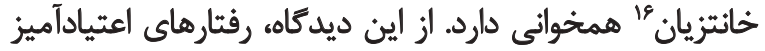

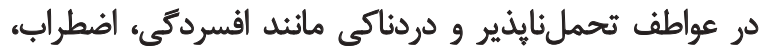

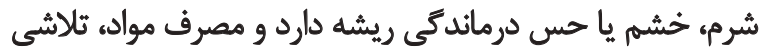

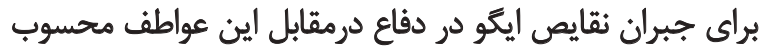

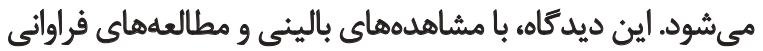

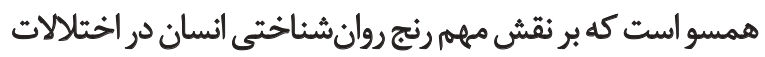

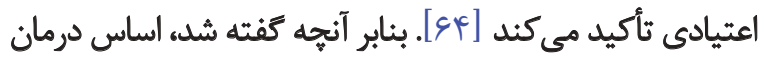

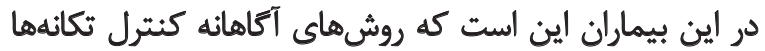

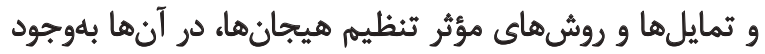

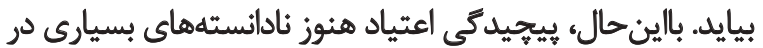

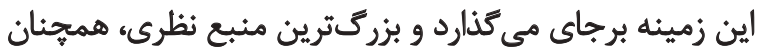
مراجعان هستند.

يكى از محدوديتهاى اين يثوهش، استفاده از آزمونهاي مداد

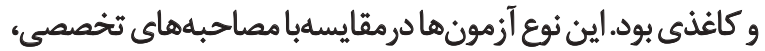

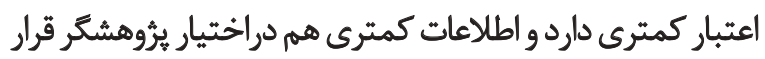

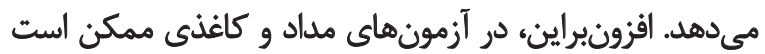

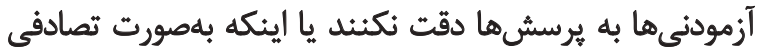

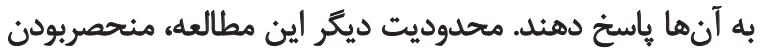

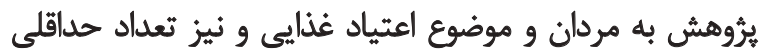

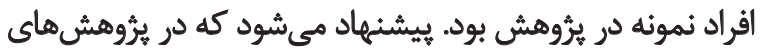

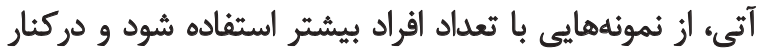

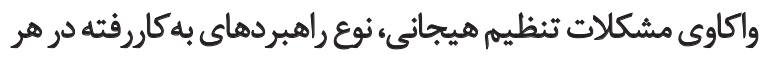

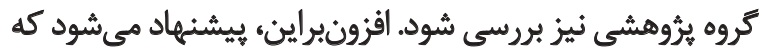

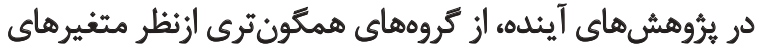

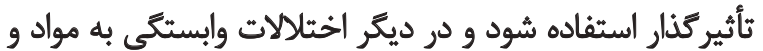

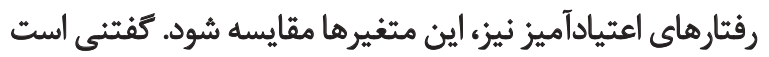

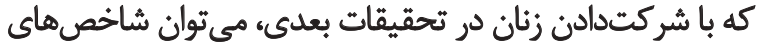

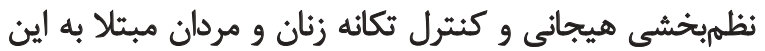
رفتارهاى اعتيادآميز را مقايسه كرد كني تكانه زنار

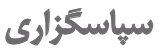

اين مقاله بركرفته از بايانانامه كارشئاسى ارشد آقاى شيرزاد

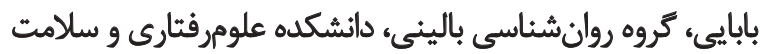

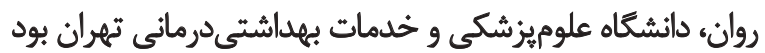

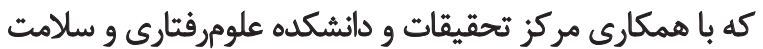

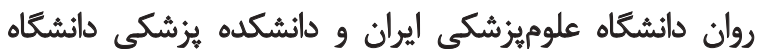

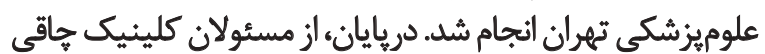
بيمارستان امامخمينى(ره) تهران و دانشجويان دريان ترامى و تمامي كاني 


\section{References}

[1] Joranby L, Pineda KF, Gold MS. Addiction to food and brain reward systems. Sexual Addiction \& Compulsivity. 2005; 12(23):201-17. doi: 10.1080/10720160500203765

[2] Merlo LJ, Klingman C, Malasanos TH, Silverstein JH. Exploration of food addiction in pediatric patients. Journal of Addiction Medicine. 2009; 3(1):26-32. doi: 10.1097/adm.0b013e31819638b0

[3] Randolph TG. The descriptive features of food addiction; addictive eating and drinking. Quarterly Journal of Studies on Alcohol. 1956; 17(2):198-224. PMID: 13336254

[4] Corwin RL, Grigson PS. Symposium overview-food addiction: fact or fiction? Journal of Nutrition. 2009; 139(3):617-9. doi: 10.3945/jn.108.097691

[5] Gearhardt AN, Yokum S, Orr PT, Stice E, Corbin WR, Brownell KD. Neural correlates of food addiction. Archives of General Psychiatry. 2011; 68(8):808. doi: 10.1001/archgenpsychiatry.2011.32

[6] Hoebel BG, Avena NM, Bocarsly ME, Rada P. A behavioral and circuit model based on sugar addiction in rats. Journal of Addiction Medicine. 2009; 3(1):33-41. doi: 10.1097/ADM.0b013e31819aa621.

[7] Avena NM, Bocarsly ME, Hoebel BG. Animal models of sugar and fat bingeing: Relationship to food addiction and increased body weight. Psychiatric Disorders. 2011; 351-65. doi: 10.1007/978-1-61779-458-2_23

[8] Gearhardt AN, White MA, Masheb RM, Grilo CM. An examination of food addiction in a racially diverse sample of obese patients with binge eating disorder in primary care settings. Comprehensive Psychiatry. 2013; 54(5):500-5. doi: 10.1016/j. comppsych.2012.12.009

[9] Gearhardt AN, Corbin WR, Brownell KD. Preliminary validation of the Yale Food Addiction Scale. Appetite. 2009; 52(2):430-6. doi: 10.1016/j.appet.2008.12.003

[10] Meule A, Vögele C, Kübler A. German translation and validation of the Yale Food Addiction Scale. Diagnostica. 2012; 58(3):115-126.

[11] American Psychiatric Association. Diagnostic and Statistical Manual of Mental Disorders, $5^{\text {th }}$ Edition: DSM-5. Washington D.C.: American Psychiatric Association; 2013.

[12] Davis C, Curtis C, Levitan RD, Carter JC, Kaplan AS, Kennedy JL. Evidence that "food addiction" is a valid phenotype of obesity. Appetite. 2011; 57(3):711-7. doi: 10.1016/j.appet.2011.08.017

[13] Spitzer RL, Yanovski S, Wadden T, Wing R, Marcus MD, Stunkard A, et al. Binge eating disorder: Its further validation in a multisite study. International Journal of Eating Disorders. 1993; 13(2):137-53. PMID: 8477283

[14] Ross HE, Ivis F. Binge eating and substance use among male and female adolescents. International Journal of Eating Disorders 1999; 26(3):245-60.

[15] Johnson PM, Kenny PJ. Dopamine D2 receptors in addictionlike reward dysfunction and compulsive eating in obese rats. Nature Neuroscience. 2010; 13(5):635-41. doi: 10.1038/nn.2519

[16] Avena NM, Rada P, Hoebel BG. Evidence for sugar addiction: Behavioral and neurochemical effects of intermittent, excessive sugar intake. Neuroscience \& Biobehavioral Reviews. 2008; 32(1):20-39. doi: 10.1016/j.neubiorev.2007.04.019
[17] Moeller FG, Barratt ES, Dougherty DM, Schmitz JM, Swann AC. Psychiatric aspects of impulsivity. American Journal of Psychiatry. 2001; 158(11):1783-93. doi: 10.1176/appi.ajp.158.11.1783

[18] Whiteside SP, Lynam DR. The five factor model and impulsivity: Using a structural model of personality to understand impulsivity. Personality and Individual Differences. 2001; 30(4):669-89. doi: 10.1016/s0191-8869(00)00064-7

[19] Adinoff B, Stein EA. Impulsivity and addiction. In: Garavan $\mathrm{H}$, editor. Neuroimaging in Addiction. New York: John Wiley \& Sons, Ltd; 2011. doi: 10.1002/9781119998938.ch7

[20] Stevens L, Verdejo-García A, Goudriaan AE, Roeyers H, Dom $G$, Vanderplasschen W. Impulsivity as a vulnerability factor for poor addiction treatment outcomes: A review of neurocognitive findings among individuals with substance use disorders. Journal of Substance Abuse Treatment. 2014; 47(1):58-72. doi: 10.1016/j jsat.2014.01.008

[21] Steiger H, Bruce KR. Phenotypes, endophenotypes, and genotypes in bulimia spectrum eating disorders. The Canadian Journal of Psychiatry. 2007; 52(4):220-7. doi: 10.1177/070674370705200403

[22] Galanti K, Gluck ME, Geliebter A. Test meal intake in obese binge eaters in relation to impulsivity and compulsivity. International Journal of Eating Disorders. 2007; 40(8):727-32. doi: 10.1002/eat.20441

[23] Verdejo-Garcia A, Benbrook A, Funderburk F, David P, Cadet JL, Bolla KI. The differential relationship between cocaine use and marijuana use on decision-making performance over repeat testing with the Iowa Gambling Task. Drug and Alcohol Dependence. 2007; 90(1):2-11. doi: 10.1016/j.drugalcdep.2007.02.004

[24] Verdejo-García A, Lawrence AJ, Clark L. Impulsivity as a vulnerability marker for substance-use disorders: Review of findings from high-risk research, problem gamblers and genetic association studies. Neuroscience \& Biobehavioral Reviews. 2008; 32(4):777-810. doi: 10.1016/j.neubiorev.2007.11.003

[25] Steel Z, Blaszczynski A. Impulsivity, personality disorders and pathological gambling severity. Addiction. 1998; 93(6):895-905. doi: $10.1046 /$ j.1360-0443.1998.93689511.x

[26] Dawe S, Loxton NJ. The role of impulsivity in the development of substance use and eating disorders. Neuroscience \& Biobehavioral Reviews. 2004; 28(3):343-51. doi: 10.1016/j.neubiorev.2004.03.007

[27] Dick DM, Smith G, Olausson P, Mitchell SH, Leeman RF, O'Malley SS, et al. Understanding the construct of impulsivity and its relationship to alcohol use disorders. Addiction Biology. 2010; 15(2):217-26. doi: 10.1111/j.1369-1600.2009.00190.x

[28] Fineberg NA, Potenza MN, Chamberlain SR, Berlin HA, Menzies L, Bechara A, et al. Probing compulsive and impulsive behaviors, from animal models to endophenotypes: A narrative review. Neuropsychopharmacology. 2009; 35(3):591-604. doi: 10.1038/ npp.2009.185

[29] Nederkoorn C, Braet C, Van Eijs Y, Tanghe A, Jansen A. Why obese children cannot resist food: The role of impulsivity. Eating Behaviors. 2006; 7(4):315-22. doi: 10.1016/j.eatbeh.2005.11.005

[30] Reisch L, Eberle U, Lorek S. Sustainable food consumption: an overview of contemporary issues and policies. Sustainability: Science, Practice, \& Policy. 2013; 9(2):7-25. 
[31] O'Leary A. Stress, emotion, and human immune function. Psychological Bulletin. 1990; 108(3):363-82. doi: 10.1037/00332909.108.3.363

[32] Carver CS, Scheier M. Principles of self-regulation: Action and emotion. In: Tory HE, Sorrentino RM, editors. Handbook of Motivation and Cognition: Foundations of Social Behavior, Vol. 2. New York: Guilford Press; 1990.

[33] Skinner BF. Science and human behavior. New York: Simon and Schuster; 1965.

[34] Toda M. Emotion and decision making. Acta Psychologica. 1980; 45(1-3):133-55. doi: 10.1016/0001-6918(80)90026-8

[35] Gross JJ, Thompson RA. Emotion regulation: Conceptual foundations. In: Gross JJ, editor. Handbook of Emotion Regulation. New York: Guilford Press; 2007.

[36] Levitan RD, Davis C. Emotions and eating behaviour: Implications for the current obesity epidemic. University of Toronto Quarterly. 2010; 79(2):783-99. doi: 10.3138/utq.79.2.783

[37] Macht M. How emotions affect eating: A five-way model. Appetite. 2008; 50(1):1-11. doi: 10.1016/j.appet.2007.07.002

[38] Brown SL, Schiraldi GR, Wrobleski PP. Association of eating behaviors and obesity with psychosocial and familial influences. American Journal of Health Education. 2009; 40(2):80-9. doi: 10.1 080/19325037.2009.10599082.

[39] Desmet PMA, Schifferstein HNJ. Sources of positive and negative emotions in food experience. Appetite. 2008; 50(2-3):290-301. doi: 10.1016/j.appet.2007.08.003

[40] Adriaanse M, de Ridder D, Evers C. Emotional eating: Eating when emotional or emotional about eating? Psychology \& Health. 2011; 26(1):23-39. doi: 10.1080/08870440903207627

[41] Gearhardt AN, White MA, Masheb RM, Morgan PT, Crosby $\mathrm{RD}$, Grilo CM. An examination of the food addiction construct in obese patients with binge eating disorder. International Journal of Eating Disorders. 2011; 45(5):657-63. doi: 10.1002/eat.20957

[42] First MB, Spitzer RL, Gibbon M, Williams JB. User's guide for the Structured clinical interview for DSM-IV axis I disorders SCID-I: Clinician version. Arlington: American Psychiatric Association Publishing; 1997.

[43] Sharifi V, Asadi SM, Mohammadi MR, Amini H, Kaviani H, Semnani Y, et al. [Reliability and feasibility of the Persian version of the structured diagnostic interview for DSM-IV (SCID) (Persian)]. Advances in Cognitive Science. 2004; 6(1,2):10-22.

[44] Flint AJ, Gearhardt AN, Corbin WR, Brownell KD, Field AE, Rimm EB. Food-addiction scale measurement in 2 cohorts of middle-aged and older women. American Journal of Clinical Nutrition. 2014; 99(3):578-86. doi: 10.3945/ajcn.113.068965

[45] Elster J. Strong feelings: Emotion, addiction, and human behavior. Cambridge, Massachusetts: MIT Press; 2012.

[46] Ekhtiari H, Safaei H, Esmaeeli Djavid GH, Atefvahid MK, Edalati $\mathrm{H}$, Mokri A. [Reliability and validity of Persian versions of Eysenck, Barratt, Dickman and Zuckerman Questionnaires in assessing risky and impulsive behaviors (Persian)]. Iranian Journal of Psychiatry and Clinical Psychology. 2008; 14(3):326-336.

[47] Gratz KL, Roemer L. Multidimensional assessment of emotion regulation and dysregulation: Development, factor structure, and initial validation of the difficulties in emotion regulation scale.
Journal of Psychopathology and Behavioral Assessment. 2004; 26(1):41-54. doi: 10.1023/b:joba.0000007455.08539.94

[48] Khanzadeh M, Saeediyan M, Hosseinchari M, Edrissi F. [Factor structure and psychometric properties of difficulties in emotional regulation scale (Persian)]. International Journal of Behavioral Sciences. 2012; 6(1):87-96.

[49] Bjork JM, Hommer DW, Grant SJ, Danube C. Impulsivity in abstinent alcohol-dependent patients: Relation to control subjects and type 1-/type 2-like traits. Alcohol. 2004; 34(2-3):133-50. doi: 10.1016/j.alcohol.2004.06.012

[50] Dom G, D'haene P, Hulstijn W, Sabbe B. Impulsivity in abstinent early- and late-onset alcoholics: Differences in self-report measures and a discounting task. Addiction. 2006; 101(1):50-9. doi: 10.1111/j.1360-0443.2005.01270.x

[51] Leland DS, Paulus MP. Increased risk-taking decision-making but not altered response to punishment in stimulant-using young adults. Drug and Alcohol Dependence. 2005; 78(1):83-90. doi: 10.1016/j.drugalcdep.2004.10.001

[52] Moeller FG, Dougherty DM. Impulsivity and substance abuse: What is the connection? Addictive Disorders \& Their Treatment. 2002; 1(1):3-10. doi: 10.1097/00132576-200205000-00002

[53] Whiteside SP, Lynam DR. Understanding the role of impulsivity and externalizing psychopathology in alcohol abuse: Application of the UPPS Impulsive Behavior Scale. Experimental and Clinical Psychopharmacology. 2003; 11(3):210-7. doi: 10.1037/1064-1297.11.3.210

[54] Bechara A, Damasio H. Decision-making and addiction (part I): impaired activation of somatic states in substance dependent individuals when pondering decisions with negative future consequences. Neuropsychologia. 2002; 40(10):1675-89. doi: 10.1016/ s0028-3932(02)00015-5

[55] Skinner MD, Aubin HJ, Berlin I. Impulsivity in smoking, nonsmoking, and ex-smoking alcoholics. Addictive Behaviors. 2004; 29(5):973-8. doi: 10.1016/j.addbeh.2004.02.045

[56] Bickel WK, Odum AL, Madden GJ. Impulsivity and cigarette smoking: Delay discounting in current, never, and ex-smokers. Psychopharmacology. 1999; 146(4):447-54. doi: 10.1007/ pl00005490

[57] Mitchell SH. Measures of impulsivity in cigarette smokers and non-smokers. Psychopharmacology. 1999; 146(4):455-64. doi: $10.1007 / \mathrm{pl} 00005491$

[58] Meule A, Heckel D, Kübler A. Factor structure and item analysis of the Yale Food Addiction Scale in obese candidates for bariatric surgery. European Eating Disorders Review. 2012; 20(5):41922. doi: $10.1002 /$ erv.2189

[59] Nederkoorn C, Braet C, Van Eijs Y, Tanghe A, Jansen A. Why obese children cannot resist food: The role of impulsivity. Eating Behaviors. 2006; 7(4):315-22. doi: 10.1016/j.eatbeh.2005.11.005

[60] Dir AL, Karyadi K, Cyders MA. The uniqueness of negative urgency as a common risk factor for self-harm behaviors, alcohol consumption, and eating problems. Addictive Behaviors. 2013; 38(5):2158-62. doi: 10.1016/j.addbeh.2013.01.025

[61] Cyders MA, Dzemidzic M, Eiler WJ, Coskunpinar A, Karyadi K, Kareken DA. Negative urgency and ventromedial prefrontal cortex responses to alcohol cues: FMRI evidence of emotionbased impulsivity. Alcoholism: Clinical and Experimental Research. 2013; 38(2):409-17. doi: 10.1111/acer.12266 
[62] Volkow ND, Wang GJ, Fowler JS, Tomasi D. Addiction circuitry in the human brain. Annual Review of Pharmacology and Toxicology. 2012; 52(1):321-36. doi: 10.1146/annurev-pharmtox-010611-134625

[63] Khantzian E, Dods LM, Brehm A. Psychodynamics. In: Lowinson HJ, Ruiz P, Millman RB, Langrod JG, editors. Substance Abuse: A Comprehensive Textbook. Philadelphia: Williams \& Wilkins; 2005.

[64] Macht M, Simons G. Emotions and eating in everyday life. Appetite. 2000; 35(1):65-71.

[65] Khantzian EJ. Addiction as a self-regulation disorder and the role of self-medication. Addiction. 2013; 108(4):668-9. doi: 10.1111/add.12004

[66] Izard CE. Human emotions. Berlin: Springer; 2013. 
